Article

\title{
An Efficient Synthetic Approach Towards Benzo[b]pyrano[2,3-e][1,4]diazepines, and Their Cytotoxic Activity
}

\author{
Islam H. El Azab 1,2,*(D) and Nadia A.A. Elkanzi 2,3 \\ 1 Chemistry Department, Faculty of Science, Taif University, Al-Haweiah, P.O. Box 888, \\ Taif 21974, Saudi Arabia \\ 2 On leave from Chemistry Department, Faculty of Science, Aswan University, Aswan, P.O. Box, \\ Aswan 81528, Egypt; kanzi20@yahoo.com \\ 3 Chemistry Department, College of Science, Jouf University, P.O. Box 2014, Sakaka, Saudi Arabia \\ * Correspondence: ihelmy2003@yahoo.com or i.helmy@tu.edu.sa; Tel.: +966-543-350-861
}

Received: 9 April 2020; Accepted: 26 April 2020; Published: 28 April 2020

\begin{abstract}
In search of unprecedented tri and/or tetrapod pharmacophoric conjugates, a series of 32 new 4-ethyl-1H-benzo[b][1,4]diazepin-2(3H)-ones were synthesized and properly elucidated using MS, IR, NMR, and elemental analysis. In vitro investigation of 11 compounds of this series, using a panel of two human tumor cell lines namely; human breast adenocarcinoma (MCF-7), and human colorectal carcinoma (HCT-116), revealed promising cytotoxic activities. Among all synthesized compounds, analogue 9 displayed maximum cytotoxicity with $\mathrm{IC}_{50}$ values of $16.19 \pm 1.35$ and $17.16 \pm 1.54 \mu \mathrm{M}$ against HCT-116 and MCF-7, respectively, compared to standard drug doxorubicin.
\end{abstract}

Keywords: cyclocondensation reaction; benzodiazepine; $N$-heterocycles; cytotoxic activity

\section{Introduction}

Benzodiazepines (BDZ's) are privileged heteroaromatic molecules and were considered to be the core of an essential class of pharmaceutically active analogues, so, their synthesis is of high value in the subject of medicinal and pharmaceutical chemistry [1-4]. 1,4-Benzodiazepines (Figure 1) are used as anti-microbial [5], in alcohol withdrawal syndrome (AWS) [6], as endothelin antagonist [7], hypnotics [8], anxiolytics [9], anticonvulsants [10], muscle relaxants [10], anticancer drugs [11,12], sedatives [13,14], and antipsychotics [15]. Recently, benzodiazepines were also recognized to have anti-proliferative [16], antimicrobial [17], anti-inflammatory [18], anti-plateletanti-ulcer [19], and analgesic [20].

Due to our admiration with the synthesis, modification, and studying the biological activities of benzodiazepines, herein, we freshened our sustained efforts [21-27], through the synthesis and utility of 5-methyl-4-(methylthio)-2-oxo-2,11-dihydrobenzo[b]pyrano[2,3-e][1,4]diazepine-3-carbonitrile (5) as a reactive precursor, for the annulation of benzopyranodiazepines of potential biological activity. 
<smiles>Clc1ccc2c(c1)C(c1ccccc1)=NCc1nncn1-2</smiles>

EStazolam<smiles>Cc1ncc2n1-c1ccc(Cl)cc1C(c1ccccc1F)=NC2</smiles>

midazolam<smiles>O=C1Nc2ccc(Cl)cc2C(c2ccccc2Cl)=NC1O</smiles>

jorazepam<smiles>O=C1CN=C(c2ccccn2)c2cc(Br)ccc2N1</smiles>

bromazepam<smiles>O=C(O)C1N=C(c2ccccc2)c2cc(Cl)ccc2NC1=O</smiles>

clorazepate<smiles>Cc1nnc2n1-c1ccc(Cl)cc1C(c1ccccc1)=NC2</smiles>

alprazolam<smiles>O=C1Nc2ccc(Cl)cc2C(c2ccccc2)=NC1O</smiles>

oxazepam<smiles>CN1C(=O)CN=C(c2ccccn2)c2cc(Cl)ccc21</smiles>

diazepam

Figure 1. Representative examples of biologically active 1,4-benzodiazepines.

\section{Results and Discussion}

\subsection{Chemistry}

In our sustained efforts to synthesize various functionalized heterocyclic analogues, and studying their biological activities [21-27], we desired to report a new efficient and simple technique for the synthesis of benzo[b]pyrano[2,3-e][1,4]diazepines. Compound $\mathbf{1}[28]$ was selected as a substrate to condense with compound 2 [29] in stirred DMSO, containing catalytic amounts of $\mathrm{NaOH}$ at $\mathrm{rt}$ to furnish pyrano[1,4]diazepine derivative 5 with an $85 \%$ yield (Scheme 1). The existence of the nitrile group was assured using its IR absorption band at $2209 \mathrm{~cm}^{-1}$ and its ${ }^{13} \mathrm{C}$ NMR as a singlet at $115.9 \mathrm{ppm}$, while the occurrence of the methylthio moiety was supported using ${ }^{1} \mathrm{H}$ NMR as a singlet at $2.73 \mathrm{ppm}$. Further, evidence for compound 5 was obtained from its noted mass at $m / z 297.06$ matches with the formula $\mathrm{C}_{15} \mathrm{H}_{11} \mathrm{~N}_{3} \mathrm{O}_{2} \mathrm{~S}$.<smiles>C[C@H]1CC(=O)Nc2ccccc2N1</smiles>

1<smiles>COC(=O)C(C#N)=C(C)S(C)(=O)=O</smiles>

2

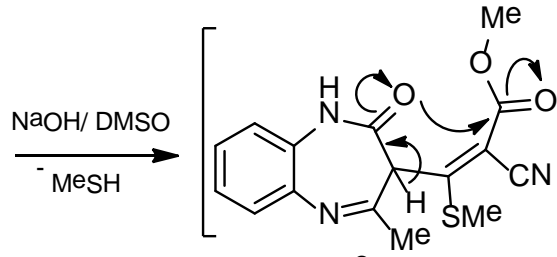

3<smiles>CCC1(OC)Nc2ccc(C)cc2NC2=C(C(C)=Nc3cc(C)ccc3N2)C(C)=C1C</smiles>

4<smiles>[3H][Mg][3H]</smiles><smiles>CC1=Nc2ccccc2Nc2oc(=O)c(C#N)c(C)c21</smiles>

Scheme 1. Synthesis of benzo[b]pyrano[2,3-e][1,4]diazepine derivative 5 . 
The good replaceable active methylthio moiety in compound 5 was cyclized with $\mathrm{NH}_{2} \mathrm{NH}_{2} \cdot \mathrm{H}_{2} \mathrm{O}$, afforded the amino pyrazole derivative 6 with a $65 \%$ yield (Scheme 2). Similarly, it was smoothly condensed with hydroxylamine, phenyl hydrazine, thiosemicarbazide, urea, thiourea, and guanidine hydrochloride to provide the target compounds (7-12). The gesture of the methylthio protons initially observed in compound $5\left({ }^{1} \mathrm{H}\right.$ NMR) at 2.73 ppm was vanished, whereas the $\mathrm{NH}_{2}$ protons were observed around $\delta \sim 7.00$ ppm.

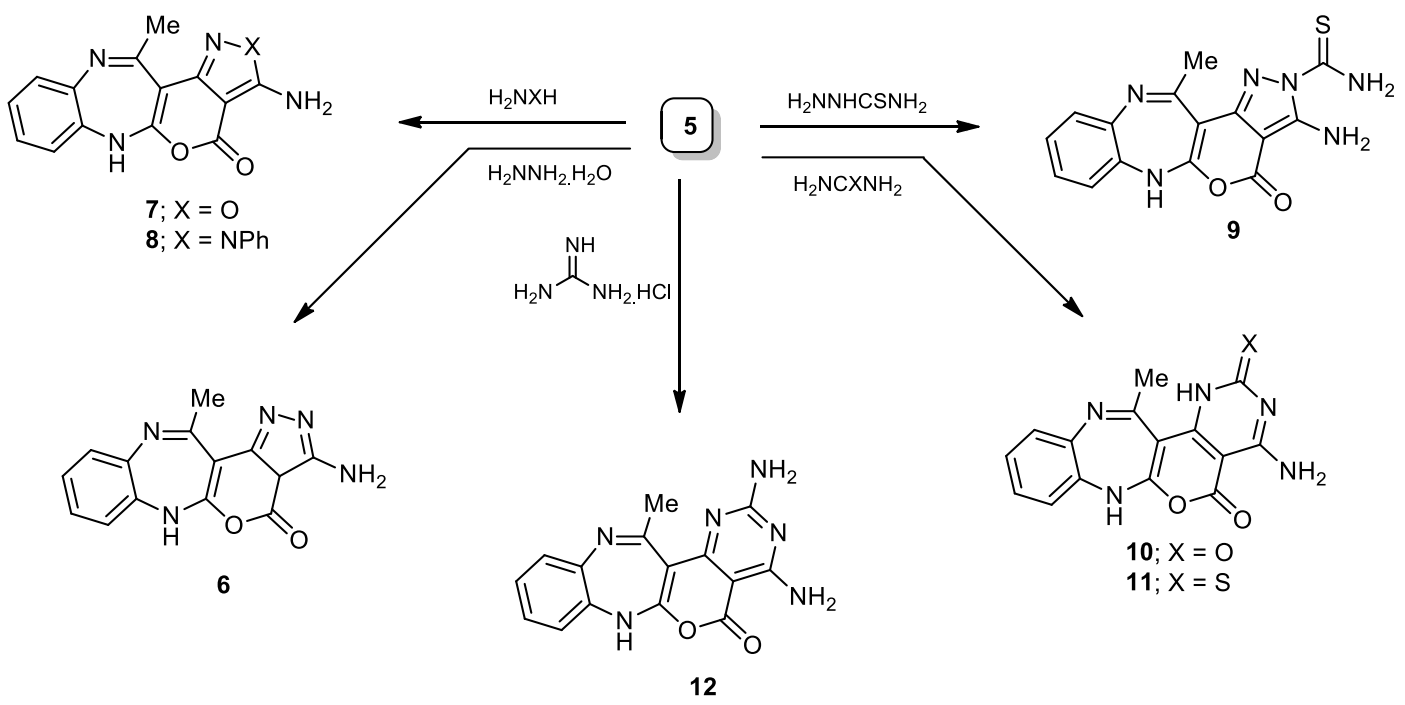

Scheme 2. Synthetic pathways of compounds 6-12.

Moreover, compound 5 was condensed with 2-aminophenol or 2-aminothiophenol, and benzo[b][1,4]oxa(thia)zepine analogues (13 and 14) were obtained (Scheme 3). The construction of compound 13 was presumed to advance via the preliminary condensation of the hydroxyl proton of 2-aminophenol and the methylthio moiety of compound 5, through removal of the methanethiol fragment, followed by further, internal cyclization to oxazepine derivative 13, through nucleophilic attack of the amino moiety onto the nitrile. The noted mass at $m / z 358.11$ matching with the formula $\mathrm{C}_{20} \mathrm{H}_{14} \mathrm{~N}_{4} \mathrm{O}_{3}$, as well the $\mathrm{NH}_{2}$ band (IR) at $\sim 3325 \mathrm{~cm}^{-1}$, and its broad singlet ( ${ }^{1} \mathrm{H} \mathrm{NMR}$ ) at $8.61 \mathrm{ppm}$, all confirmed structure 13. Upon treatment of compound 5 with tris(hydroxymethyl)aminomethane or $\mathrm{PhNH}_{2}$, the secondary amine analogues (15 and 16) were obtained. Compound 15 (IR) showed interest bands centered at 2217 and $3406 \mathrm{~cm}^{-1}$, owing to the hydroxyl and the nitrile groups, correspondingly, while, its ${ }^{1} \mathrm{H}$ NMR scope exhibited three new singlets at 3.02, 3.81, and 5.74 ppm, owing to the hydroxyl, methylene, and the secondary amine protons, respectively. In a similar manner, compound 5 was subjected to some selected secondary amines namely; diethylamine, morpholine, $N$-methylpiperazine and/or piperazinyl to furnish the corresponding tertiary amines (17-20). Strong absorption sign centered around $2210 \mathrm{~cm}^{-1}$ owing to the nitrile group, supported these structures. Compound 17 ( ${ }^{1} \mathrm{H}$ NMR) demonstrated a triplet and quartet signs allocated to the two equivalent ethyl moieties at 1.16 and 3.35 ppm, respectively. Furthermore, compound 5 was cyclocondensed with cyanothioacetamide, afforded 1,3-thiazine analogue 21 with an $80 \%$ yield (Scheme 3). The cyanomethyl and imino protons in compound 21 appeared as two singlets ( ${ }^{1} \mathrm{H}$ NMR) at 4.15 and 8.91 ppm, respectively. 


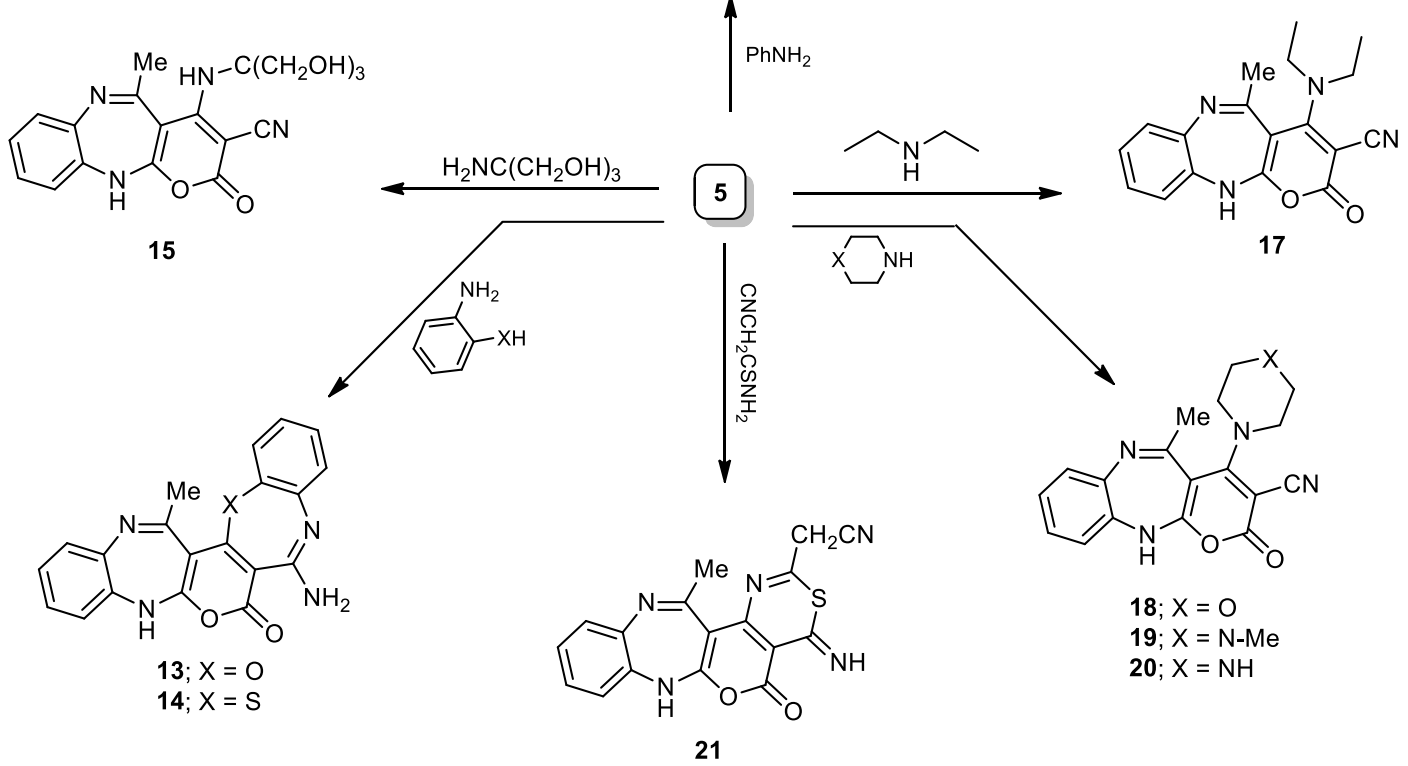

Scheme 3. Synthetic pathways of compounds 13-21.

Similarly, upon smooth cyclocondensation of compound $\mathbf{5}$ with some heterocyclic amines, a series of ploy fused heterocyclic systems (22-28) were acquired (Scheme 4).<smiles>NC1=Nc2ccccc2NC(=O)c2c1nn1c(=S)[nH]c(=O)cc1c2N</smiles>

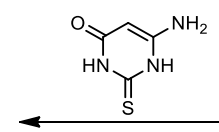

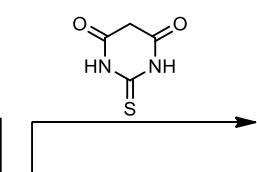<smiles></smiles>

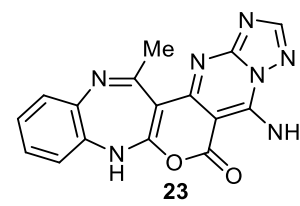<smiles></smiles><smiles></smiles>

22<smiles>Nc1nc2ccccc2[nH]c1=O</smiles>

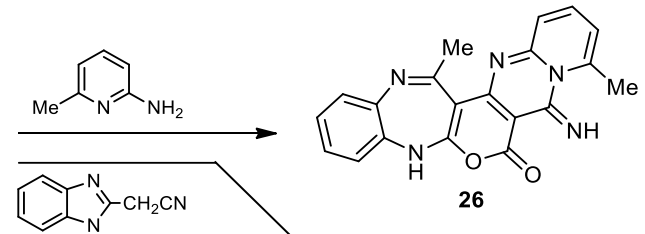

28

Scheme 4. Synthetic pathways of compounds 22-28. 
The adaptability of our heteroaromatic construction policy was spare, as demonstrated by the annulation of pyrano[c]pyran analogue. Thus, cyclocondensation of intermediate 5 with cyclopentanone or acetylacetone, afforded the pyrano[c]pyran derivatives (32 and 33), respectively (Scheme 5). The formation of compound 31 could be supposed to advance through the preliminary condensation of the energetic methylene in the cyclopentanone with the easily removable methylthio moiety in compound 5, to afford the non-isolable intermediates 29 and 30, which underwent internal cyclization through a nucleophilic attack of the $\mathrm{OH}$ to the $\mathrm{C} \equiv \mathrm{N}$ fragment, to furnish the final product 31. The exocyclic imino moiety in compound 31, was easily converted to carbonyl via treatment with $\mathrm{HCl}$ in boiling $\mathrm{EtOH}$, to afford cyclopenta[b]pyran-2-one derivative 32 (Scheme 5). Compound 32 (IR) showed intense bands at 1705, $3256 \mathrm{~cm}^{-1}$ owing to the carbonyl and imino moieties, correspondingly, whereas its mass spectrum presented a molecular ion peak $\left(\mathrm{C}_{19} \mathrm{H}_{14} \mathrm{~N}_{2} \mathrm{O}_{4}\right)$ at $m / z 334.10$.

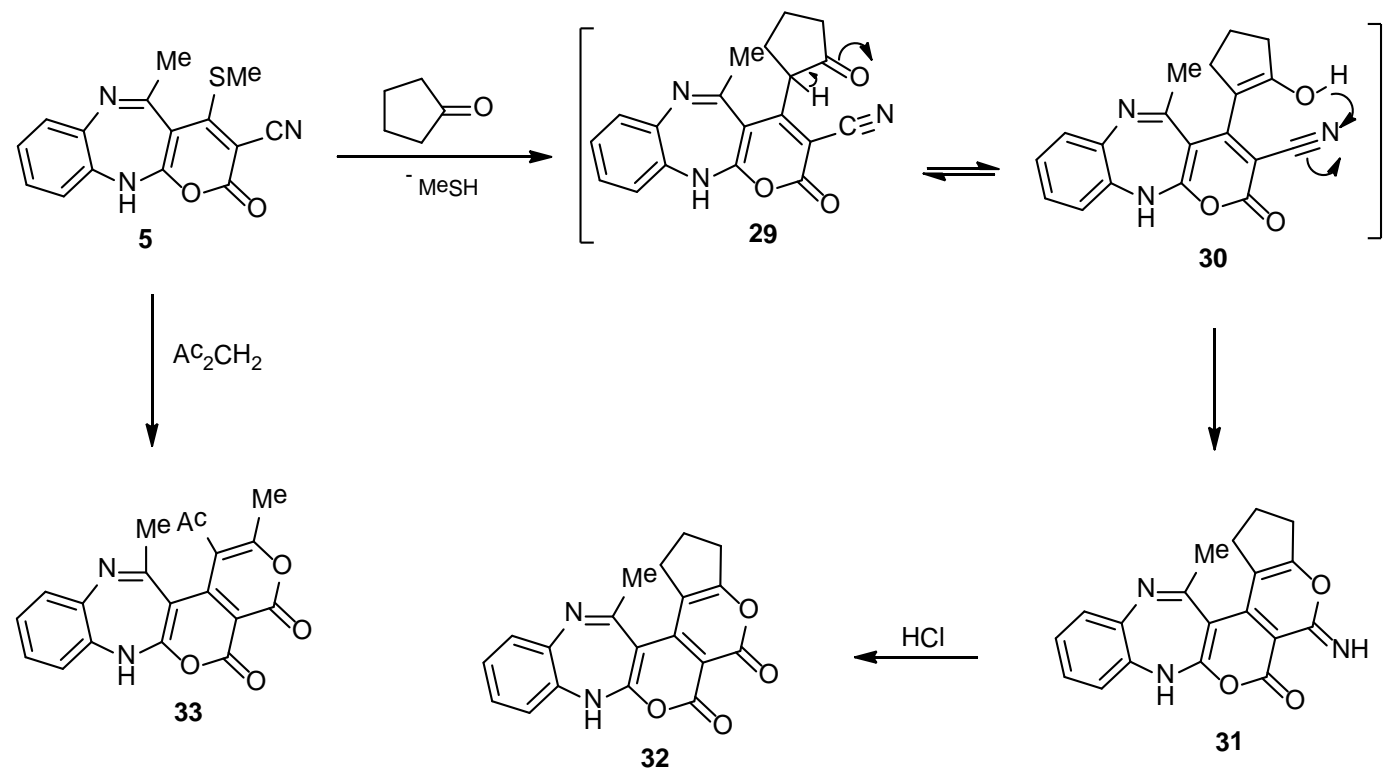

Scheme 5. Synthetic pathways of compounds 31-33.

Finally, $\alpha$-aminocarbothiamide analogue 9 was applied as a facile point to construct the target pyrazolo[1,5-a][1,3,5]triazine-4-thiones (34-38) (Scheme 6), through smooth condensation with triethyl orthoformate, acetyl chloride, ethyl cyanoacetate, chloroacetyl chloride, and carbon disulfide. The mass spectrum of compound 36 presented an ion peak at $m / z 389.05\left(\mathrm{C}_{19} \mathrm{H}_{14} \mathrm{~N}_{2} \mathrm{O}_{4}\right)$, whereas its IR declared two distinctive bands owing to the $\mathrm{C}=\mathrm{S}$ and $\mathrm{C} \equiv \mathrm{N}$ moieties, at 1325 and $2215 \mathrm{~cm}^{-1}$ correspondingly.<smiles>CC1=Nc2ccccc2Nc2oc(=O)c3c(N)n(C(N)=S)nc3c21</smiles>

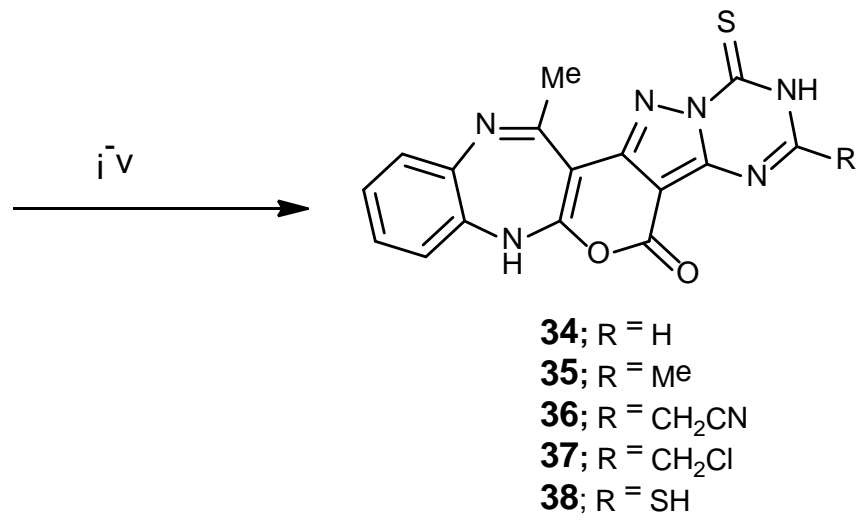

Scheme 6. Reagents and conditions for synthesis of compounds 34-38. Note: $\mathrm{i}=\mathrm{HC}(\mathrm{OEt})_{3} ; \mathrm{ii}=\mathrm{AcCl}$; iii $=\mathrm{CNCH}_{2} \mathrm{CO}_{2} \mathrm{Et}$; and iv $=\mathrm{ClCOCH}_{2} \mathrm{Cl} ; \mathrm{v}=\mathrm{CS}_{2} / \mathrm{EtOH}$. 


\subsection{Pharmacological Evaluation}

\section{Cytotoxic Impact}

According to the Sulforhodamine B (SRB) method [30,31], new eleven conjugates were in vitro examined for their cytotoxic impact towards human colorectal carcinoma (HCT-116) and human breast adenocarcinoma (MCF-7). Doxorubicin was the positive drug, while DMSO was the negative control. The cytotoxic impacts of the investigated compounds are presented in Table 1.

Table 1. Preliminary in vitro cytotoxicity values of some new synthesized compounds.

\begin{tabular}{ccc}
\hline \multirow{2}{*}{ Compound No. } & \multicolumn{2}{c}{ Cytotoxic Effect $\left(\mathrm{IC}_{\mathbf{5 0}}(\boldsymbol{\mu M})\right) \pm \mathrm{SD}$} \\
\cline { 2 - 3 } & MCF-7 & HCT-116 \\
\hline $\mathbf{5}$ & $95.26 \pm 2.32$ & $90.91 \pm 3.21$ \\
$\mathbf{6}$ & $19.48 \pm 0.15$ & $18.78 \pm 1.02$ \\
$\mathbf{7}$ & $17.54 \pm 1.20$ & $17.20 \pm 1.12$ \\
$\mathbf{8}$ & $22.18 \pm 0.98$ & $21.97 \pm 0.76$ \\
$\mathbf{9}$ & $17.16 \pm 1.54$ & $16.19 \pm 1.35$ \\
$\mathbf{1 5}$ & $24.54 \pm 1.87$ & $24.11 \pm 2.13$ \\
$\mathbf{1 6}$ & $33.54 \pm 1.42$ & $32.54 \pm 1.28$ \\
$\mathbf{1 7}$ & $82.02 \pm 0.95$ & $81.18 \pm 0.32$ \\
$\mathbf{1 8}$ & $46.76 \pm 1.27$ & $45.73 \pm 0.87$ \\
$\mathbf{1 9}$ & $56.24 \pm 2.20$ & $55.41 \pm 0.35$ \\
$\mathbf{2 0}$ & $60.00 \pm 1.25$ & $58.81 \pm 1.28$ \\
Doxorubicin & $10.34 \pm 0.23$ & $9.88 \pm 0.15$
\end{tabular}

Values in the range 1-20 indicate 'very strong', 21-40 indicate 'strong', 41-60 indicate 'moderate', and 61-100 indicate 'very weak'. IC 50 is the concentration of compound that is required to decrease the feasibility of the cells by 50\%, compared to the non-treated control cells. MCF-7: Human breast tumor cell line; HCT-116: human colorectal carcinoma; and doxorubicin (Adriamycin): Positive control compound.

Analyses of the $\mathrm{IC}_{50}$ data as given in Table 1 declare that, majority of the investigated compounds own noteworthy cytotoxic activities versus these cell lines. Where, compound 9 was the most effective among the screened series with $\mathrm{IC}_{50}=16.19 \pm 1.35$ and $17.16 \pm 1.54 \mu \mathrm{M}$ against HCT-116 and MCF-7, respectively. Moreover, the test cell lines were generally susceptible to conjugates 7, 6, 8, 15, 16, 18, 19, and 20, in a downward order, with $\mathrm{IC}_{50}<60.00 \mu \mathrm{M}$. Extra reading of the obtained results stated that, compounds 5 and 17 were less robust towards the two tumor cell lines with $\mathrm{IC}_{50}>60.00 \mu \mathrm{M}$.

In conclusion, a series of 32 new benzo[ $b][1,4]$ diazepines was synthesized as a bipod or tripod pharmacophoric architectures that could reinforce the cytotoxic impact. The presence of the parent skeleton benzo-pyrano-diazepine was vital for the extensive spectrum of cytotoxic action against the screened cell lines. Moreover, introducing the pyrazole ring bearing carbothioamide fragment to the parent skeleton enhanced the anti-tumor ability of compound 9 to become close to the doxorubicin. The cytotoxic activity of compounds 7, 6, and 8 was attributed to the presence of the amino oxazole as well as amino pyrazole moieties, in conjugation with the parent benzo-pyrano-diazepine skeleton. Whereas introduction of 1,1,1-tri hydroxymethyl methyl amine moiety in compound 15, enforced it to show an excellent potency; moreover, the existence of a phenylamino fragment in compound 16 improved the molecule to show a strong cytotoxicity. On the other hand, introducing the piperazinyl or morpholinyl moiety in compounds 17-20 minified the potency of these molecules, compared to the other tested compounds. 


\section{Materials and Methods}

\subsection{General Information}

Reagents were purchased from Sigma Aldrich (Bayouni Trading Co. Ltd., Al-Khobar, Saudi Arabia) and used without further purification. The reaction progress was monitored by TLC on silica gel pre-coated F254 Merck plates (Merck, Darmstadt, Germany). Spots were visualized by ultraviolet irradiation. Melting points were determined on a digital Gallen-Kamp MFB-595 instrument (Gallenkamp, London, UK) using open capillary tubes and were uncorrected. IR spectra were recorded as potassium bromide discs using Bruker-Vector 22 FTIR spectrophotometer (Bruker, Manasquan, NJ, USA). The NMR spectra were recorded with a Varian Mercury VXR-300 NMR spectrometer (Bruker, Marietta, GA, USA) at 300 and $75 \mathrm{MHz}$ for ${ }^{1} \mathrm{H}$ and ${ }^{13} \mathrm{C}$ NMR spectra, respectively, using DMSO- $d_{6}$ as the solvent. Mass spectra were recorded on a Hewlett Packard MS-5988 spectrometer (Hewlett Packard, Palo Alto, CA, USA) at 70 eV. Elemental analyses were conducted at the Micro-Analytical Center of Taif University, Taif, KSA. The pharmaceutical activity assays were carried out at the applied research sector, Egyptian company for vaccine and serum (VACSERA, Cairo, Egypt).

5-Methyl-4-(methylthio)-2-oxo-2,11-dihydrobenzo[b]pyrano[2,3-e][1,4]diazepine-3-carbonitrile

Compound 1 [28] (0.17 g, $1 \mathrm{mmol})$, methyl 2-cyano-3,3-dimethylthioacrylate [29] (0.20 mg, $1 \mathrm{mmol})$ and powdered $\mathrm{KOH}(0.08 \mathrm{~g}, 1.5 \mathrm{mmol})$ in DMSO $(25 \mathrm{~mL})$, were stirred at $\mathrm{rt}$ for $3 \mathrm{~h}$. The reaction mixture was transferred onto mashed ice under energetic stirring for $1 \mathrm{~h}$. The isolated product was collected, dried, and recrystallized using EtOH to furnish 5 as a pale yellow solid, with an 85\% yield; $\operatorname{mp} 149-151{ }^{\circ} \mathrm{C}$; IR (KBr): $\left(\mathrm{cm}^{-1}\right) 1612\left(\mathrm{C}=\mathrm{N}_{\text {str. }}\right), 1705\left(\mathrm{C}=\mathrm{O}_{\text {str. }}\right), 2209\left(\mathrm{C} \mathrm{N}_{\text {str. }}\right), 3206\left(\mathrm{~N}-\mathrm{H}_{\text {str. }}\right){ }^{1}{ }^{1} \mathrm{H}$ NMR

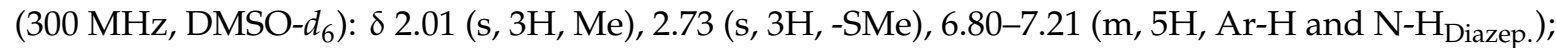
${ }^{13} \mathrm{C}$ NMR (75 MHz, DMSO- $\left.d_{6}\right):$ 15.3, $25.6(2 \mathrm{Me}), 115.9(\mathrm{C} \equiv \mathrm{N}), 76.8,86.4,113.6,123.5,124.1,126.6,128.5$, 137.3, 138.1 (9 C=C), 149.5 (C=O), $164.5(\mathrm{C}=\mathrm{N}), 179.8$ (=C-S-); MS ( $m / z, \%): 297.06\left(\mathrm{M}^{+}, 35\right)$; Anal. Calcd. for $\mathrm{C}_{15} \mathrm{H}_{11} \mathrm{~N}_{3} \mathrm{O}_{2} \mathrm{~S}$ (297.33): C, 60.59; H, 3.73; N, 14.13\%. Found: $\mathrm{C}, 60.21 ; \mathrm{H}, 3.35 ; \mathrm{N}, 14.01 \%$.

General procedure of the synthesis of compounds (6-28). Compound $5(0.29 \mathrm{~g}, 1 \mathrm{mmol})$ and some selected amino compounds namely; ethylenediamine, hydrazine hydrate, hydroxylamine hydrochloride, phenyl hydrazine, thiosemicarbazide, urea, thiourea, guanidine hydrochloride, 2-aminophenol, 2-aminothiophenol, 2-amino-2-(hydroxymethyl)propane-1,3-diol, aniline, secondary amines, 2-cyanothioacetamide, 5-amino-3-phenyl-1H-pyrazole, 6-amino-2-thioxo-2,3dihydropyrimidin-4(1H)-one, 3-amino-1,2,4- triazole, 2-thioxodihydropyrimidine-4,6(1H,5H)-dione, 6-methylpyridin-2-amine, 2-cyanomethyl benzimidazole, and 2-amino benzimidazole in $25 \mathrm{~mL}$ of $\mathrm{EtOH}$ has $\mathrm{Et}_{3} \mathrm{~N}(0.3 \mathrm{~mL})$, was refluxed for 7-10 h and the reaction advance was checked by TLC. After evaporation of $\mathrm{EtOH}$, the crude product was triturated with acetone, and the isolated solid was filtered and purified by crystallization, using the proper solvent to furnish compounds (6-28) in fair yields.

3-Amino-12-methyl-1H-benzo[b]pyrazolo[3', $\left.4^{\prime}: 4,5\right]$ pyrano[2,3-e][1,4]diazepin-4(6H)-one (6). Yellow powder $(\mathrm{EtOH})$ with a 65\% yield; mp 205-207 ${ }^{\circ} \mathrm{C}$; IR $(\mathrm{KBr}):\left(\mathrm{cm}^{-1}\right) 1610-1623\left(3 \mathrm{C}=\mathrm{N}_{\text {str. }}\right), 1705\left(\mathrm{C}=\mathrm{O}_{\text {str. }}\right)$, 3206-3325 (N-H str., N-H 2 str. $) ;{ }^{1} \mathrm{H}$ NMR (300 MHz, DMSO-d $): \delta 2.01$ (s, 3H, Me), $2.51\left(\mathrm{~s}, 1 \mathrm{H}, \mathrm{Pyran}_{(\mathrm{C} 3)}-\mathrm{H}\right)$,

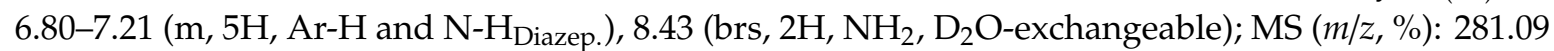
$\left(\mathrm{M}^{+}, 15\right)$; Anal. Calcd. for $\mathrm{C}_{14} \mathrm{H}_{11} \mathrm{~N}_{5} \mathrm{O}_{2}$ (281.27): C, 59.78; H, 3.94; N, 24.90\%. Found: C, 59.47; H, 3.63; $\mathrm{N}, 24.59 \%$.

3-Amino-12-methylbenzo[b]isoxazolo[3',4':4,5]pyrano[2,3-e][1,4]diazepin-4(6H)-one (7). Yellow crystal $(\mathrm{EtOH})$ with a $71 \%$ yield; mp 241-243 ${ }^{\circ} \mathrm{C}$; IR (KBr): $\left(\mathrm{cm}^{-1}\right) 1610-1623\left(2 \mathrm{C}=\mathrm{N}_{\text {str. }}\right), 1705\left(\mathrm{C}=\mathrm{O}_{\text {str. }}\right)$, 3206-3325 (N-H str., N-H 2 str.); ${ }^{1} \mathrm{H} \mathrm{NMR} \mathrm{(300} \mathrm{MHz,} \mathrm{DMSO-d} 6$ ): $\delta 2.01$ (s, 3H, Me), 6.80-7.21 (m, 7H, Ar-H, N-H Diazep. and $\left.\mathrm{NH}_{2}\right)$; $\mathrm{MS}(\mathrm{m} / \mathrm{z}, \%)$ : $282.07\left(\mathrm{M}^{+}, 50\right)$; Anal. Calcd. for $\mathrm{C}_{14} \mathrm{H}_{10} \mathrm{~N}_{4} \mathrm{O}_{3}$ (282.25): C, 59.57; H, 3.57; N, 19.85\%. Found: C, 59.31; H, 3.20; N, 19.52\%. 
3-Amino-12-methyl-2-phenyl-2H-benzo[b]pyrazolo $\left[3^{\prime}, 4^{\prime}: 4,5\right]$ pyrano[2,3-e][1,4]diazepin-4(6H)-one (8). Yellow solid $(\mathrm{MeOH})$ with a $65 \%$ yield; $\mathrm{mp} 223-225^{\circ} \mathrm{C}$; IR $(\mathrm{KBr}):\left(\mathrm{cm}^{-1}\right) 1610-1623\left(2 \mathrm{C}=\mathrm{N}_{\text {str. }}\right), 1705\left(\mathrm{C}=\mathrm{O}_{\text {str }}\right)$, 3206-3325 (N-H str., $\left.\mathrm{N}-\mathrm{H}_{2 s t r .}\right) ;{ }^{1} \mathrm{H}$ NMR (300 MHz, DMSO-d $\left.d_{6}\right): \delta 2.01(\mathrm{~s}, 3 \mathrm{H}, \mathrm{Me}), 6.80-7.21(\mathrm{~m}, 13 \mathrm{H}$, Ar-H, N-H $\mathrm{H}_{\text {Diazep. }}$ and $\left.\mathrm{NH}_{2}\right)$; $\mathrm{MS}(\mathrm{m} / \mathrm{z}, \%)$ : $357.12\left(\mathrm{M}^{+}, 15\right)$; Anal. Calcd. for $\mathrm{C}_{20} \mathrm{H}_{15} \mathrm{~N}_{5} \mathrm{O}_{2}$ (357.37): $\mathrm{C}$, 67.22; H, 4.23; N, 19.60\%. Found: C, 67.12; H, 4.10; N, 19.42\%.

3-Amino-12-methyl-4-oxo-4,6-dihydro-2H-benzo[b]pyrazolo [3', $\left.4^{\prime}: 4,5\right]$ pyrano[2,3-e][1,4]diazepine-2carbothioamide (9). Pale-yellow powder $(\mathrm{EtOH})$ with a $75 \%$ yield; mp $143-145^{\circ} \mathrm{C}$; IR $(\mathrm{KBr}):\left(\mathrm{cm}^{-1}\right)$ $1325\left(\mathrm{C}=\mathrm{S}_{\text {str. }}\right), 1610-1623\left(2 \mathrm{C}=\mathrm{N}_{\text {str. }}\right), 1705\left(\mathrm{C}=\mathrm{O}_{\text {str. }}\right), 3206-3325\left(\mathrm{~N}-\mathrm{H}_{\text {str. }}, \mathrm{N}-\mathrm{H}_{2 s t r}\right){ }^{2}{ }^{1} \mathrm{H} \mathrm{NMR}(300 \mathrm{MHz}$, DMSO- $\left.d_{6}\right): \delta 2.01(\mathrm{~s}, 3 \mathrm{H}, \mathrm{Me}), 6.80-7.21\left(\mathrm{~m}, 7 \mathrm{H}, \mathrm{Ar}-\mathrm{H}, \mathrm{N}-\mathrm{H}_{\text {Diazep. }}\right.$ and $\left.\mathrm{NH}_{2}\right), 8.53$ (brs, $2 \mathrm{H}, \mathrm{CSNH}_{2}$, $\mathrm{D}_{2} \mathrm{O}$-exchangeable); $\mathrm{MS}(\mathrm{m} / \mathrm{z}, \%): 340.07\left(\mathrm{M}^{+}, 25\right)$; Anal. Calcd. for $\mathrm{C}_{15} \mathrm{H}_{12} \mathrm{~N}_{6} \mathrm{O}_{2} \mathrm{~S}$ (340.36): $\mathrm{C}, 52.93 ; \mathrm{H}$, $3.55 ; \mathrm{N}, 24.69 \%$. Found: C, 52.71; H, 3.26; N, 24.35\%.

4-Amino-13-methylbenzo[b]pyrimido[4', $\left.5^{\prime}: 4,5\right]$ pyrano[2,3-e][1,4]diazepine-2,5(1H,7H)-dione (10). Yellowishbrown solid (EtOH) with an $82 \%$ yield; mp $165-167^{\circ} \mathrm{C}$; IR $(\mathrm{KBr}):\left(\mathrm{cm}^{-1}\right) 1327\left(\mathrm{C}=\mathrm{S}_{\text {str. }}\right), 1610-1623$ $\left(2 \mathrm{C}=\mathrm{N}_{\text {str. }}\right), 1705-1715\left(2 \mathrm{C}=\mathrm{O}_{\text {str. }}\right), 3206-3325\left(\mathrm{~N}-\mathrm{H}_{\text {str. }}, \mathrm{N}-\mathrm{H}_{2 s t r}\right) ;{ }^{1} \mathrm{H} \mathrm{NMR}\left(300 \mathrm{MHz}, \mathrm{DMSO}-d_{6}\right): \delta 2.01$ (s, 3H, Me), 6.80-7.21 (m, 5H, Ar-H and N-H Diazep.), 8.61 (brs, $2 \mathrm{H}, \mathrm{NH}_{2}, \mathrm{D}_{2} \mathrm{O}$-exchangeable), 12.51 (brs, $\mathrm{H}, \mathrm{NH}_{\text {Pyrim }}$, $\mathrm{D}_{2} \mathrm{O}$-exchangeable); ${ }^{13} \mathrm{C}$ NMR $\left(75 \mathrm{MHz}\right.$, DMSO-d $\left.d_{6}\right): 25.6(\mathrm{Me}), 76.8,91.5,113.6,123.5$, 124.1, 126.6, 128.5, 137.3, 138.1, $152.5(9 \mathrm{C}=\mathrm{C}), 156.2,162.7(2 \mathrm{C}=\mathrm{O}), 164.5,167.1(2 \mathrm{C}=\mathrm{N})$; $\mathrm{MS}(\mathrm{m} / \mathrm{z}, \%)$ : $310.01\left(\mathrm{M}^{+}+1,40\right)$; Anal. Calcd. for $\mathrm{C}_{15} \mathrm{H}_{11} \mathrm{~N}_{5} \mathrm{O}_{3}$ (309.28): $\mathrm{C}, 58.25 ; \mathrm{H}, 3.58 ; \mathrm{N}, 22.64 \%$. Found: $\mathrm{C}, 58.04$; $\mathrm{H}, 3.21 ; \mathrm{N}, 22.34 \%$.

4-Amino-13-methyl-2-thioxo-1,2-dihydrobenzo[b]pyrimido[4', 5':4,5]pyrano[2,3-e][1,4]diazepin-5(7H)-one (11). Yellow crystals $(\mathrm{MeOH})$ with an $80 \%$ yield; $\mathrm{mp} 205-207^{\circ} \mathrm{C}$; IR $(\mathrm{KBr}):\left(\mathrm{cm}^{-1}\right) 1321\left(\mathrm{C}=\mathrm{S}_{\text {str. }}\right), 1610-1623$ $\left(2 \mathrm{C}=\mathrm{N}_{\text {str. }}\right), 1705\left(\mathrm{C}=\mathrm{O}_{\text {str. }}\right), 3206-3325\left(\mathrm{~N}-\mathrm{H}_{\text {str. }}, \mathrm{N}-\mathrm{H}_{2 s t r}\right) ;{ }^{1} \mathrm{H}$ NMR $\left(300 \mathrm{MHz}, \mathrm{DMSO}-d_{6}\right): \delta 2.01(\mathrm{~s}, 3 \mathrm{H}$,

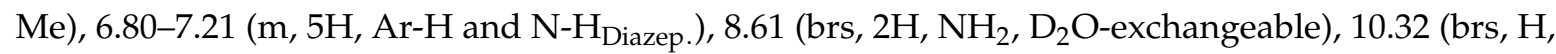
$\mathrm{NH}_{\text {Pyrim., }} \mathrm{D}_{2} \mathrm{O}$-exchangeable); $\mathrm{MS}(\mathrm{m} / \mathrm{z}, \%)$ : $325.06\left(\mathrm{M}^{+}, 55\right)$; Anal. Calcd. for $\mathrm{C}_{15} \mathrm{H}_{11} \mathrm{~N}_{5} \mathrm{O}_{2} \mathrm{~S}(325.35)$ : C, 55.38; H, 3.41; N, 21.53\%. Found: C, 55.02; H, 3.15; N, 21.24\%.

2,4-Diamino-13-methylbenzo[b]pyrimido[4',5':4,5]pyrano[2,3-e][1,4]diazepin-5(7H)-one (12). Yellow crystals $(\mathrm{EtOH})$ with a $75 \%$ yield; mp $221-223{ }^{\circ} \mathrm{C}$; IR $(\mathrm{KBr}):\left(\mathrm{cm}^{-1}\right) 1610-1623\left(2 \mathrm{C}=\mathrm{N}_{\text {str. }}\right), 1705\left(\mathrm{C}=\mathrm{O}_{\text {str. }}\right)$, 3206-3325 (N-H str. $\left._{2} 2 \mathrm{~N}-\mathrm{H}_{2 s t r}\right) ;{ }^{1} \mathrm{H}$ NMR (300 MHz, DMSO-d $\left.d_{6}\right): \delta 2.01(\mathrm{~s}, 3 \mathrm{H}, \mathrm{Me}), 6.80-7.21(\mathrm{~m}, 9 \mathrm{H}$, Ar-H, N-H Diazep. and $2 \mathrm{NH}_{2}$.); $\mathrm{MS}(\mathrm{m} / \mathrm{z}, \%): 308.10\left(\mathrm{M}^{+}, 30\right)$; Anal. Calcd. for $\mathrm{C}_{15} \mathrm{H}_{12} \mathrm{~N}_{6} \mathrm{O}_{2}$ (308.29): $\mathrm{C}$, 58.44; H, 3.92; N, 27.26\%. Found: C, 58.21; H, 3.65; N, 27.11\%.

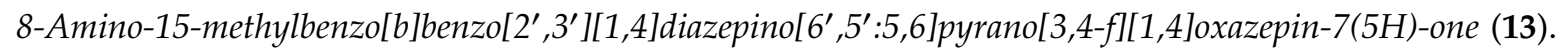
Off-white powder (EtOH) with a $65 \%$ yield; $\mathrm{mp} 241-243^{\circ} \mathrm{C}$; IR (KBr): $\left(\mathrm{cm}^{-1}\right) 1610-1623\left(2 \mathrm{C}=\mathrm{N}_{\text {str }}\right), 1705$ $\left(\mathrm{C}=\mathrm{O}_{\text {str. }}\right), 3206-3325\left(\mathrm{~N}-\mathrm{H}_{\text {str. }}, \mathrm{N}-\mathrm{H}_{2 s t r}\right) ;{ }^{1} \mathrm{H}$ NMR $\left(300 \mathrm{MHz}, \mathrm{DMSO}-d_{6}\right): \delta 2.01(\mathrm{~s}, 3 \mathrm{H}, \mathrm{Me}), 6.80-7.21(\mathrm{~m}$,

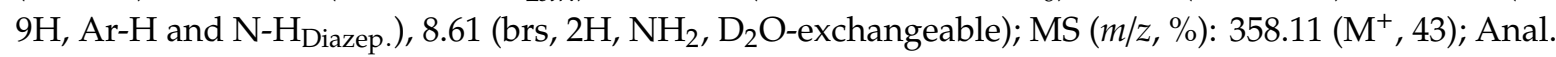
Calcd. for $\mathrm{C}_{20} \mathrm{H}_{14} \mathrm{~N}_{4} \mathrm{O}_{3}$ (358.35): C, 67.03; H, 3.94; N, 15.63\%. Found: $\mathrm{C}, 66.86 ; \mathrm{H}, 3.67 ; \mathrm{N}, 15.42 \%$.

8-Amino-15-methylbenzo[b]benzo[2' $\left.{ }^{\prime} 3^{\prime}\right][1,4]$ diazepino[ $\left.6^{\prime}, 5^{\prime}: 5,6\right]$ pyrano[3,4-f][1,4]thiazepin-7(5H)-one (14). Yellow crystals (MeOH/dioxane (2:1)) with a $75 \%$ yield; mp 176-178 ${ }^{\circ} \mathrm{C}$; IR (KBr): $\left(\mathrm{cm}^{-1}\right)$ 1610-1623 $\left(2 \mathrm{C}=\mathrm{N}_{\text {str. }}\right), 1705\left(\mathrm{C}=\mathrm{O}_{\text {str. }}\right), 3206-3325\left(\mathrm{~N}-\mathrm{H}_{\text {str. }}, \mathrm{N}-\mathrm{H}_{2 s t r .}\right) ;{ }^{1} \mathrm{H} \mathrm{NMR}\left(300 \mathrm{MHz}, \mathrm{DMSO}-d_{6}\right): \delta 2.01(\mathrm{~s}, 3 \mathrm{H}$, $\mathrm{Me}), 6.80-7.21$ (m, 9H, Ar-H and N-H $\left.\mathrm{H}_{\text {Diazep. }}\right), 8.60$ (brs, $2 \mathrm{H}, \mathrm{NH}_{2}, \mathrm{D}_{2} \mathrm{O}-$ exchangeable); $\mathrm{MS}(\mathrm{m} / \mathrm{z}, \%)$ : $374.08\left(\mathrm{M}^{+}\right.$, 15); Anal. Calcd. for $\mathrm{C}_{20} \mathrm{H}_{14} \mathrm{~N}_{4} \mathrm{O}_{2} \mathrm{~S}$ (374.42): $\mathrm{C}, 64.16 ; \mathrm{H}, 3.77 ; \mathrm{N}, 14.96 \%$. Found: $\mathrm{C}, 64.01$; $\mathrm{H}, 3.51 ; \mathrm{N}, 14.58 \%$.

4-((1,3-Dihydroxy-2-(hydroxymethyl)propan-2-yl)amino)-5-methyl-2-oxo-2,11-dihydrobenzo[b]pyrano[2,3-e] [1,4]diazepine-3-carbonitrile (15). Yellowish-brown solid (MeOH) with a $75 \%$ yield; $\mathrm{mp} 215-217^{\circ} \mathrm{C}$; IR $(\mathrm{KBr}):\left(\mathrm{cm}^{-1}\right) 1615\left(\mathrm{C}=\mathrm{N}_{\text {str. }}\right), 1705\left(\mathrm{C}=\mathrm{O}_{\text {str. }}\right), 2217\left(\mathrm{C} \equiv \mathrm{N}_{\text {str. }}\right), 3226\left(2 \mathrm{~N}-\mathrm{H}_{s t r}\right), 3406\left(3{\mathrm{O}-\mathrm{H}_{\text {str }}}\right) ;{ }^{1} \mathrm{H} \mathrm{NMR}$ (300 MHz, DMSO- $\left.d_{6}\right): \delta 2.01(\mathrm{~s}, 3 \mathrm{H}, \mathrm{Me}), 3.20\left(\mathrm{~s}, 6 \mathrm{H}, 3 \mathrm{CH}_{2}\right), 3.61$ (brs, $3 \mathrm{H}, 3 \mathrm{OH}, \mathrm{D}_{2} \mathrm{O}-$ exchangeable), 4.02 (brs, $1 \mathrm{H}, \mathrm{NH}, \mathrm{D}_{2} \mathrm{O}$-exchangeable), 6.80-7.21 (m, 5H, Ar-H and $\mathrm{N}-\mathrm{H}_{\text {Diazep }}$ )) ${ }^{13} \mathrm{C} \mathrm{NMR}(75 \mathrm{MHz}$, DMSO- $\left.d_{6}\right): 26.3(\mathrm{Me}), 63.4\left(3 \mathrm{CH}_{2}\right), 114.6(\mathrm{C} \equiv \mathrm{N}), 63.5,74.9(4 \mathrm{C}-\mathrm{C}), 63.3,76.8,113.5,123.5,124.1,126.6$, 
128.5, 137.3, 138.1, $181.9(10 \mathrm{C}=\mathrm{C}), 162.3(\mathrm{C}=\mathrm{O}), 164.5(\mathrm{C}=\mathrm{N})$; $\mathrm{MS}(\mathrm{m} / \mathrm{z}, \%)$ : $370.13\left(\mathrm{M}^{+}, 25\right)$; Anal. Calcd. for $\mathrm{C}_{18} \mathrm{H}_{18} \mathrm{~N}_{4} \mathrm{O}_{5}$ (370.36): C, 58.37; H, 4.90; N, 15.13\%. Found: C, 58.12; H, 4.74; N, 15.02\%.

5-Methyl-2-oxo-4-(phenylamino)-2,11-dihydrobenzo[b]pyrano[2,3-e][1,4]diazepine-3-carbonitrile (16). White powder $(\mathrm{EtOH})$ with an $80 \%$ yield; $\mathrm{mp} 232-234{ }^{\circ} \mathrm{C}$; IR $(\mathrm{KBr}):\left(\mathrm{cm}^{-1}\right) 1612\left(\mathrm{C}=\mathrm{N}_{\text {str. }}\right), 1705\left(\mathrm{C}=\mathrm{O}_{\text {str. }}\right), 2209$ $\left(\mathrm{C} \equiv \mathrm{N}_{s t r}\right), 3206\left(2 \mathrm{~N}-\mathrm{H}_{\text {str. }}\right) ;{ }^{1} \mathrm{H}$ NMR $\left(300 \mathrm{MHz}, \mathrm{DMSO}-d_{6}\right): \delta 2.01(\mathrm{~s}, 3 \mathrm{H}, \mathrm{Me}), 6.80-7.21(\mathrm{~m}, 10 \mathrm{H}, \mathrm{Ar}-\mathrm{H}$ and $\left.\mathrm{N}-\mathrm{H}_{\text {Diazep. }}\right), 8.71$ (brs, $1 \mathrm{H}, \mathrm{NH}, \mathrm{D}_{2} \mathrm{O}$-exchangeable); $\mathrm{MS}(\mathrm{m} / \mathrm{z}, \%): 342.10\left(\mathrm{M}^{+}, 10\right)$; Anal. Calcd. for $\mathrm{C}_{20} \mathrm{H}_{14} \mathrm{~N}_{4} \mathrm{O}_{2}$ (342.35): C, 70.17; H, 4.12; N, 16.37\%. Found: C, 70.01; H, 4.02; N, 16.13\%.

4-(Diethylamino)-5-methyl-2-oxo-2,11-dihydrobenzo[b]pyrano[2,3-e][1,4]diazepine-3-carbonitrile (17). Light brown solid (EtOH) with a $65 \%$ yield; mp $182-184^{\circ} \mathrm{C}$; IR $(\mathrm{KBr}):\left(\mathrm{cm}^{-1}\right) 1612\left(\mathrm{C}=\mathrm{N}_{\text {str. }}\right), 1705\left(\mathrm{C}=\mathrm{O}_{\text {str. }}\right)$, $2209\left(\mathrm{C} \equiv \mathrm{N}_{s t r .}\right), 3206\left(\mathrm{~N}-\mathrm{H}_{s t r .}\right) ;{ }^{1} \mathrm{H}$ NMR $\left(300 \mathrm{MHz}, \mathrm{DMSO}-d_{6}\right): \delta 1.16\left(\mathrm{t}, 6 \mathrm{H}, 2 \mathrm{CH}_{2} \mathrm{CH}_{3}\right), 2.01(\mathrm{~s}, 3 \mathrm{H}, \mathrm{Me})$, 3.35 (q, $\left.4 \mathrm{H}, 2 \mathrm{CH}_{2} \mathrm{CH}_{3}\right), 6.80-7.21$ (m, 5H, Ar-H and N-H Diazep.); $\mathrm{MS}(\mathrm{m} / \mathrm{z}, \%): 322.14\left(\mathrm{M}^{+}, 25\right) ;$ Anal. Calcd. for $\mathrm{C}_{18} \mathrm{H}_{18} \mathrm{~N}_{4} \mathrm{O}_{2}$ (322.36): C, 67.07; H, 5.63; N, 17.38\%. Found: $\mathrm{C}, 66.86 ; \mathrm{H}, 5.35 ; \mathrm{N}, 17.14 \%$.

5-Methyl-4-morpholino-2-oxo-2,11-dihydrobenzo[b]pyrano[2,3-e][1,4]diazepine-3-carbonitrile (18). Buff solid (EtOH) with a 70\% yield; mp 142-144 ${ }^{\circ} \mathrm{C}$; IR ( $\left.\mathrm{KBr}\right):\left(\mathrm{cm}^{-1}\right) 1612\left(\mathrm{C}=\mathrm{N}_{\text {str. }}\right), 1705\left(\mathrm{C}=\mathrm{O}_{\text {str. }}\right), 2209\left(\mathrm{C} \equiv \mathrm{N}_{\text {str. }}\right)$, $3206\left(\mathrm{~N}-\mathrm{H}_{\text {str. }}\right) ;{ }^{1} \mathrm{H}$ NMR $\left(300 \mathrm{MHz}, \mathrm{DMSO}-d_{6}\right): \delta 2.01$ (s, 3H, Me), $3.64\left(\mathrm{t}, 4 \mathrm{H}\right.$, Morpho.(C2),(C6) $\left.-\mathrm{H}_{4}\right), 3.83$ (m, 4H, Morpho.(C3),(C5) $\left.{ }^{-H_{4}}\right), 6.80-7.21\left(\mathrm{~m}, 5 \mathrm{H}, \mathrm{Ar}-\mathrm{H}\right.$ and $\left.\mathrm{N}-\mathrm{H}_{\text {Diazep. }}\right) ;{ }^{13} \mathrm{C}$ NMR (75 MHz, DMSO- $\left.d_{6}\right)$ : $26.1(\mathrm{Me}), 52.4,67.3\left(4 \mathrm{CH}_{2}\right), 114.6(\mathrm{C} \equiv \mathrm{N}), 59.3,76.7,113.5,123.5,124.1,126.6,128.5,137.3,138.1,184.9$ $(10 \mathrm{C}=\mathrm{C}), 162.3(\mathrm{C}=\mathrm{O}), 164.5(\mathrm{C}=\mathrm{N})$; $\mathrm{MS}(\mathrm{m} / \mathrm{z}, \%): 336.12\left(\mathrm{M}^{+}, 9\right)$; Anal. Calcd. for $\mathrm{C}_{18} \mathrm{H}_{16} \mathrm{~N}_{4} \mathrm{O}_{3}(336.34)$ : C, 64.28; H, 4.79; N, 16.66\%. Found C, 64.10; H, 4.43; N, 16.28\%.

5-Methyl-4-(4-methylpiperazin-1-yl)-2-oxo-2,11-dihydrobenzo[b]pyrano[2,3-e][1,4]diazepine-3-carbonitrile (19). Buff solid (EtOH) with a 75\% yield; mp 193-195 ${ }^{\circ} \mathrm{C}$; IR (KBr): $\left(\mathrm{cm}^{-1}\right) 1612\left(\mathrm{C}=\mathrm{N}_{\text {str. }}\right), 1705\left(\mathrm{C}=\mathrm{O}_{\text {str. }}\right)$, $2209\left(\mathrm{C} \equiv \mathrm{N}_{s t r}\right), 3206\left(\mathrm{~N}-\mathrm{H}_{s t r .}\right) ;{ }^{1} \mathrm{H}$ NMR $\left(300 \mathrm{MHz}, \mathrm{DMSO}-d_{6}\right): \delta 2.01(\mathrm{~s}, 3 \mathrm{H}, \mathrm{Me}), 2.21(\mathrm{~s}, 3 \mathrm{H}, \mathrm{Me}), 2.32$ (t, 4H, Piperaz.(C2),(C6) $\left.-\mathrm{H}_{4}\right), 2.76\left(\mathrm{t}, 4 \mathrm{H}\right.$, Piperaz.(C3),(C5) $\left.-\mathrm{H}_{4}\right), 6.80-7.21\left(\mathrm{~m}, 5 \mathrm{H}, \mathrm{Ar}-\mathrm{H}\right.$ and $\left.\mathrm{N}-\mathrm{H}_{\text {Diazep. }}\right)$; MS (m/z, \%): $349.15\left(\mathrm{M}^{+}, 15\right)$; Anal. Calcd. for $\mathrm{C}_{19} \mathrm{H}_{19} \mathrm{~N}_{5} \mathrm{O}_{2}$ (349.39): $\mathrm{C}, 65.32 ; \mathrm{H}, 5.48 ; \mathrm{N}, 20.04 \%$. Found C, 65.11; H, 5.23; N, 19.81\%.

5-Methyl-2-oxo-4-(piperazin-1-yl)-2,11-dihydrobenzo[b]pyrano[2,3-e][1,4]diazepine-3-carbonitrile (20). Offwhite powder (EtOH) with an 80\% yield; mp 219-221 ${ }^{\circ} \mathrm{C}$; IR (KBr): $\left(\mathrm{cm}^{-1}\right) 1612\left(\mathrm{C}=\mathrm{N}_{\text {str }}\right), 1705\left(\mathrm{C}=\mathrm{O}_{\text {str. }}\right)$, $2209\left(\mathrm{C} \equiv \mathrm{N}_{\text {str. }}\right), 3206-3211\left(2 \mathrm{~N}-\mathrm{H}_{s t r}\right) ;{ }^{1} \mathrm{H}$ NMR $\left(300 \mathrm{MHz}, \mathrm{DMSO}-d_{6}\right): \delta 2.32\left(\mathrm{t}, 4 \mathrm{H}\right.$, Piperaz.(C2),(C6) $\left.-\mathrm{H}_{4}\right)$, $2.76\left(\mathrm{t}, 4 \mathrm{H}\right.$, Piperaz.(C3),(C5) $\left.-\mathrm{H}_{4}\right), 3.01$ (s, $\left.1 \mathrm{H}, \mathrm{N}-\mathrm{H}_{\text {Piperaz. }}\right), 6.80-7.21$ (m, 5H, Ar-H and N-H $\left.\mathrm{H}_{\text {Diazep. }}\right)$; $\mathrm{MS}$ $(\mathrm{m} / \mathrm{z}, \%): 335.10\left(\mathrm{M}^{+}, 30\right)$; Anal. Calcd. for $\mathrm{C}_{18} \mathrm{H}_{17} \mathrm{~N}_{5} \mathrm{O}_{2}$ (335.36): $\mathrm{C}, 64.47 ; \mathrm{H}, 5.11 ; \mathrm{N}, 20.88 \%$. Found $\mathrm{C}$, $64.25 ; \mathrm{H}, 5.01 ; \mathrm{N}, 20.52 \%$.

2-(4-Imino-13-methyl-5-oxo-5,7-dihydro-4H-[1,3]thiazino[ $\left.4^{\prime}, 5^{\prime}: 4,5\right]$ pyrano[2,3-e]benzo[b][1,4]diazepin-2-yl) acetonitrile (21). Yellow solid (MeOH/dioxane (2:1)) with an 80\% yield; mp 206-208 ${ }^{\circ} \mathrm{C}$; $\mathrm{IR}(\mathrm{KBr})$ : $\left(\mathrm{cm}^{-1}\right) 1610-1623\left(2 \mathrm{C}=\mathrm{N}_{\text {str }}\right), 1705\left(\mathrm{C}=\mathrm{O}_{\text {str. }}\right), 2209\left(\mathrm{C} \equiv \mathrm{N}_{\text {str. }}\right), 3206-3325\left(2 \mathrm{~N}-\mathrm{H}_{\text {str. }}\right) ;{ }^{1} \mathrm{H} \mathrm{NMR}(300 \mathrm{MHz}$, DMSO- $\left.d_{6}\right): \delta 2.01$ (s, 3H, Me), $4.15\left(\mathrm{~s}, 2 \mathrm{H}, \mathrm{CH}_{2}\right), 6.80-7.21$ (m, 9H, Ar-H and N-H $\left.\mathrm{H}_{\text {Diazep. }}\right), 8.91$ (brs, $\mathrm{H}$, $=\mathrm{NH}, \mathrm{D}_{2} \mathrm{O}$-exchangeable); $\mathrm{MS}(\mathrm{m} / \mathrm{z}, \%): 349.06\left(\mathrm{M}^{+}, 45\right)$; Anal. Calcd. for $\mathrm{C}_{17} \mathrm{H}_{11} \mathrm{~N}_{5} \mathrm{O}_{2} \mathrm{~S}$ (349.37): $\mathrm{C}$, 58.44; H, 3.17; N, 20.05\%. Found: C, 58.20; H, 3.01; N, 19.76\%.

5-Amino-14-methyl-2-phenylbenzo[b]pyrazolo[1", 5": $\left.1^{\prime}, 2^{\prime}\right]$ pyrimido[4',5':4,5]pyrano[2,3-e][1,4]diazepin-6(8H)one (22). Pale yellow crystalline solid (EtOH) with an $85 \%$ yield; mp $228-230{ }^{\circ} \mathrm{C}$; IR $(\mathrm{KBr}):\left(\mathrm{cm}^{-1}\right)$ 1610-1623 $\left(3 \mathrm{C}=\mathrm{N}_{\text {str. }}\right), 1705\left(\mathrm{C}=\mathrm{O}_{s t r}\right), 3206-3325\left(\mathrm{~N}-\mathrm{H}_{s t r}, \mathrm{~N}-\mathrm{H}_{2 s t r .}\right) ;{ }^{1} \mathrm{H}$ NMR $\left(300 \mathrm{MHz}, \mathrm{DMSO}-d_{6}\right): \delta$ 2.01 (s, 3H, Me), 6.54 (s, 1H, Pyraz.(C4)-H), 6.80-8.03 (m, 12H, Ar-H, N-H Diazep. and $\mathrm{NH}_{2}$.); $\mathrm{MS}(\mathrm{m} / \mathrm{z}, \%)$ : $408.13\left(\mathrm{M}^{+}, 10\right)$; Anal. Calcd. for $\mathrm{C}_{23} \mathrm{H}_{16} \mathrm{~N}_{6} \mathrm{O}_{2}$ (408.41): $\mathrm{C}, 67.64 ; \mathrm{H}, 3.95 ; \mathrm{N}, 20.58 \%$. Found: $\mathrm{C}, 67.32$; $\mathrm{H}, 3.68 ; \mathrm{N}, 20.26 \%$.

5-Amino-14-methyl-[1,2,4]triazolo[1", 5": $\left.1^{\prime}, 2^{\prime}\right]$ pyrimido[4', 5':4,5]pyrano[2,3-e]benzo[b][1,4]diazepin-6(8H)-one (23). Pale yellow powder solid (EtOH) with a $72 \%$ yield; $\mathrm{mp} 241-243{ }^{\circ} \mathrm{C}$; IR (KBr): $\left(\mathrm{cm}^{-1}\right) 1610-1623$ $\left(4 \mathrm{C}=\mathrm{N}_{s t r}\right), 1705\left(\mathrm{C}=\mathrm{O}_{\text {str. }}\right), 3206-3325\left(\mathrm{~N}-\mathrm{H}_{\text {str. }},{\left.\mathrm{N}-\mathrm{H}_{2 s t r}\right)}\right){ }^{1} \mathrm{H} \mathrm{NMR}\left(300 \mathrm{MHz}, \mathrm{DMSO}-d_{6}\right): \delta 2.01(\mathrm{~s}, 3 \mathrm{H}$, $\mathrm{Me}), 6.80-7.23$ (m, 5H, Ar-H and N-H $\mathrm{H}_{\text {Diazep. }}$ ), 7.81 (brs, 2H, NH $\mathrm{N}_{2}, \mathrm{D}_{2} \mathrm{O}$-exchangeable), 8.65 (s, 1H, 
Triaz.(C3)-H); ${ }^{13} \mathrm{C}$ NMR (75 MHz, DMSO-d $\left.d_{6}\right): 19.8$ (Me), 83.9, 107.3, 113.5, 123.5, 124.1, 126.5, 128.5, 138.2, 139.1, 168.6 (9 C=C), $150.3(\mathrm{C}=\mathrm{O}), 155.2,156.1,164.5,166.1(4 \mathrm{C}=\mathrm{N}) ; \mathrm{MS}(\mathrm{m} / \mathrm{z}, \%): 333.09\left(\mathrm{M}^{+}, 55\right)$; Anal. Calcd. for $\mathrm{C}_{16} \mathrm{H}_{11} \mathrm{~N}_{7} \mathrm{O}_{2}$ (333.30): C, 57.66; H, 3.33; N, 29.42\%. Found: C, 57.39; H, 3.13; N, 29.19\%. 6-Amino-15-methyl-4-thioxo-3,4-dihydro-2H-benzo[b]pyrimido[1", $\left.6^{\prime \prime}: 1^{\prime}, 2^{\prime}\right]$ pyrimido $\left[4^{\prime}, 5^{\prime}: 4,5\right]$ pyrano[2,3-e] [1,4]diazepine-2,7(9H)-dione (24). Light brown crystalline solid (EtOH) with an 80\% yield; $\mathrm{mp}$ 195-197 ${ }^{\circ} \mathrm{C}$; IR (KBr): $\left(\mathrm{cm}^{-1}\right) 1321\left(\mathrm{C}=\mathrm{S}_{\text {str. }}\right), 1610-1623\left(2 \mathrm{C}=\mathrm{N}_{\text {str. }}\right), 1705-1715\left(2 \mathrm{C}=\mathrm{O}_{\text {str. }}\right), 3206-3325\left(2 \mathrm{~N}-\mathrm{H}_{\text {str. }}\right.$, $\left.\mathrm{N}-\mathrm{H}_{2 s t r}\right) ;{ }^{1} \mathrm{H}$ NMR $\left(300 \mathrm{MHz}, \mathrm{DMSO}-d_{6}\right): \delta 2.06(\mathrm{~s}, 3 \mathrm{H}, \mathrm{Me}), 6.80-7.21$ (m, 5H, Ar-H and N-H $\left.\mathrm{H}_{\text {Diazep. }}\right)$, 8.52 (brs, $2 \mathrm{H}, \mathrm{NH}_{2}, \mathrm{D}_{2} \mathrm{O}$-exchangeable), 9.64 (brs, $\mathrm{H}, \mathrm{NH}_{\text {Pyrim., }} \mathrm{D}_{2} \mathrm{O}$-exchangeable); $\mathrm{MS}(\mathrm{m} / \mathrm{z}, \%$ ): $392.08\left(\mathrm{M}^{+}, 20\right)$; Anal. Calcd. for $\mathrm{C}_{18} \mathrm{H}_{12} \mathrm{~N}_{6} \mathrm{O}_{3} \mathrm{~S}$ (392.39): C, 55.10; $\mathrm{H}, 3.08 ; \mathrm{N}, 21.42 \%$. Found: $\mathrm{C}, 55.02$; $\mathrm{H}, 3.10 ; \mathrm{N}, 21.19 \%$.

6-Imino-15-methyl-3-thioxo-3,4,6,9-tetrahydro-1H-benzo[b]pyrimido[5", $\left.4^{\prime \prime}: 5^{\prime}, 6^{\prime}\right]$ pyrano $\left[4^{\prime}, 3^{\prime}: 4,5\right]$ pyrano [2,3-e][1,4]diazepine-1,7(2H)-dione (25). Brown solid (EtOH) with a $75 \%$ yield; $\mathrm{mp} 165-167^{\circ} \mathrm{C}$; $\mathrm{IR}(\mathrm{KBr})$ : $\left(\mathrm{cm}^{-1}\right) 1321\left(\mathrm{C}=\mathrm{S}_{\text {str. }}\right), 1610-1623\left(2 \mathrm{C}=\mathrm{N}_{\text {str. }}\right), 1705-1715\left(2 \mathrm{C}=\mathrm{O}_{\text {str }}\right), 3206-3325\left(4 \mathrm{~N}-\mathrm{H}_{\text {str. }}\right) ;{ }^{1} \mathrm{H}$ NMR $(300$ MHz, DMSO- $d_{6}$ ): $\delta 2.06$ (s, 3H, Me), 6.80-7.21 (m, 5H, Ar-H and N-H Diazep.), 8.68 (brs, $\mathrm{H},=\mathrm{NH}$, $\mathrm{D}_{2} \mathrm{O}$-exchangeable), 9.64, 13.21 (brs, $2 \mathrm{H}, 2 \mathrm{NH}_{\text {Pyrim. }}$, $\mathrm{D}_{2} \mathrm{O}$-exchangeable); $\mathrm{MS}(\mathrm{m} / \mathrm{z}, \%): 393.05\left(\mathrm{M}^{+}, 60\right)$; Anal. Calcd. for $\mathrm{C}_{18} \mathrm{H}_{11} \mathrm{~N}_{5} \mathrm{O}_{4} \mathrm{~S}$ (393.38): C, 54.96; H, 2.82; N, 17.80\%. Found: C, 54.65; H, 2.42; N, $17.58 \%$.

8-Imino-10,15-dimethyl-5H-benzo[b]pyrido[1",2": $\left.1^{\prime}, 2^{\prime}\right]$ pyrimido $\left[4^{\prime}, 5^{\prime}: 4,5\right]$ pyrano[2,3-e][1,4]diazepin-7(8H)-one (26). Orange powder (MeOH/DMF (3:1)) with a $75 \%$ yield; $\mathrm{mp}>300^{\circ} \mathrm{C}$; IR (KBr): $\left(\mathrm{cm}^{-1}\right) 1610-1623$ $\left(3 \mathrm{C}=\mathrm{N}_{\text {str. }}\right), 1705\left(\mathrm{C}=\mathrm{O}_{\text {str. }}\right), 3206-3325\left(2 \mathrm{~N}-\mathrm{H}_{\text {str. }}\right) ;{ }^{1} \mathrm{H} \mathrm{NMR}\left(300 \mathrm{MHz}, \mathrm{DMSO}-d_{6}\right): \delta 2.06,2.75(\mathrm{~s}, 6 \mathrm{H}$, 2Me), 6.80-7.21 (m, 8H, Ar-H, N-H Diazep. and Pyrid. $(\mathrm{C} 3,4,5)-3 \mathrm{H}), 8.92$ (brs, $\mathrm{H},=\mathrm{NH}, \mathrm{D}_{2} \mathrm{O}-$ exchangeable); ${ }^{13} \mathrm{C}$ NMR (75 MHz, DMSO- $\left.d_{6}\right): 25.2,26.1$ (2Me), 76.6, 108.2, 113.5, 115.5, 123.5, 124.1, 125.1, 126.5, 128.5, 134.1, 137.2, 138.2, 156.8, $159.6(14 \mathrm{C}=\mathrm{C}), 162.7(\mathrm{C}=\mathrm{O}), 150.5,158.9,164.5(3 \mathrm{C}=\mathrm{N})$; $\mathrm{MS}(\mathrm{m} / \mathrm{z}, \%): 357.12$ $\left(\mathrm{M}^{+}, 50\right)$; Anal. Calcd. for $\mathrm{C}_{20} \mathrm{H}_{15} \mathrm{~N}_{5} \mathrm{O}_{2}$ (357.37): $\mathrm{C}, 67.22 ; \mathrm{H}, 4.23 ; \mathrm{N}, 19.60 \%$. Found: $\mathrm{C}, 67.10 ; \mathrm{H}, 4.11$; $\mathrm{N}, 19.34 \%$.

8-Amino-16-methyl-7-oxo-5,7-dihydrobenzo[b]benzo[4",5"]imidazo[1", 2":1',6']pyrido[4',3': 4,5]pyrano[2,3-e] [1,4]diazepine-15-carbonitrile (27). Yellowish-brown powder $(\mathrm{EtOH})$ with an $80 \%$ yield; $\mathrm{mp} 215-217^{\circ} \mathrm{C}$; IR (KBr): $\left(\mathrm{cm}^{-1}\right)$ 1610-1623 (2C=N $\left.\mathrm{N}_{\text {str }}\right), 1705\left(\mathrm{C}=\mathrm{O}_{\text {str. }}\right), 2210\left(\mathrm{C} \equiv \mathrm{N}_{\text {str. }}\right), 3206-3325\left(\mathrm{~N}-\mathrm{H}_{\text {str. }}, \mathrm{N}-\mathrm{H}_{2 s t r}\right){ }^{1} \mathrm{H}$ NMR (300 MHz, DMSO- $\left.d_{6}\right): \delta 2.07$ (s, 3H, Me), 6.51 (brs, $2 \mathrm{H}, \mathrm{NH}_{2}, \mathrm{D}_{2} \mathrm{O}$-exchangeable), 6.80-8.03 (m, $9 \mathrm{H}, \mathrm{Ar}-\mathrm{H}$ and $\left.\mathrm{N}-\mathrm{H}_{\text {Diazep. }}\right) ; \mathrm{MS}(\mathrm{m} / \mathrm{z}, \%): 406.12\left(\mathrm{M}^{+}, 15\right)$; Anal. Calcd. for $\mathrm{C}_{23} \mathrm{H}_{14} \mathrm{~N}_{6} \mathrm{O}_{2}$ (406.40): $\mathrm{C}$, 67.97; H, 3.47; N, 20.68\%. Found: C, 67.61; H, 3.27; N, 20.38\%.

8-Amino-16-methylbenzo[b]benzo[4",5"]imidazo[1",2": $\left.1^{\prime}, 2^{\prime}\right]$ pyrimido[4', 5':4,5]pyrano[2,3-e][1,4]diazepin7(5H)-one (28). Yellow powder (MeOH/DMF (3:1)) with a $75 \%$ yield; mp $175-177^{\circ} \mathrm{C}$; IR ( $\left.\mathrm{KBr}\right):\left(\mathrm{cm}^{-1}\right)$ 1610-1623 (3C= $\left.\mathrm{N}_{s t r}\right), 1705\left(\mathrm{C}=\mathrm{O}_{s t r}\right), 3206-3325\left(\mathrm{~N}-\mathrm{H}_{s t r}, \mathrm{~N}-\mathrm{H}_{2 s t r}\right) ;{ }^{1} \mathrm{H}$ NMR $\left(300 \mathrm{MHz}, \mathrm{DMSO}-d_{6}\right): \delta$ 2.07 (s, 3H, Me), 6.54 (brs, 2H, NH ${ }_{2}, \mathrm{D}_{2} \mathrm{O}-$-exchangeable), 6.80-8.45 (m, 9H, Ar-H and N-H $\mathrm{H}_{\text {Diazep. }}$ ); MS ( $m / z, \%): 382.13\left(\mathrm{M}^{+}, 60\right)$; Anal. Calcd. for $\mathrm{C}_{21} \mathrm{H}_{14} \mathrm{~N}_{6} \mathrm{O}_{2}$ (382.37): $\mathrm{C}, 65.96 ; \mathrm{H}, 3.69 ; \mathrm{N}, 21.98 \%$. Found: C, 65.68; H, 3.38; N, 21.67\%.

5-Imino-14-methyl-2,3,5,8-tetrahydrobenzo[b]cyclopenta[5' $\left.6^{\prime}\right]$ pyrano[ $\left.4^{\prime}, 3^{\prime}: 4,5\right]$ pyrano[2,3-e][1,4]diazepin6(1H)-one (31). A solution of $5(0.29 \mathrm{~g}, 1 \mathrm{mmol})$ and cyclopentanone $(0.08 \mathrm{~g}, 1 \mathrm{mmol})$ in ethanolic piperidine solution $(30 \mathrm{~mL})$ was refluxed for $7 \mathrm{~h}$, afterwards the reaction mixture was left to cool to rt, the obtained precipitate was filtered, dried, and recrystallized using $\mathrm{EtOH}$ to afford 31 as a brown solid with a $65 \%$ yield, $\mathrm{mp} 268-270{ }^{\circ} \mathrm{C}$; IR $(\mathrm{KBr}):\left(\mathrm{cm}^{-1}\right) 1610\left(\mathrm{C}=\mathrm{N}_{\text {str. }}\right), 1705\left(\mathrm{C}=\mathrm{O}_{\text {str. }}\right), 3206-3325$ $\left(2 \mathrm{~N}-\mathrm{H}_{\text {str. }}\right) ;{ }^{1} \mathrm{H}$ NMR $\left(300 \mathrm{MHz}, \mathrm{DMSO}-d_{6}\right): \delta 1.83\left(\mathrm{~m}, 2 \mathrm{H}, \mathrm{CH}_{2}\right), 2.06(\mathrm{~s}, 3 \mathrm{H}, \mathrm{Me}), 2.43\left(\mathrm{t}, 2 \mathrm{H}, \mathrm{CH}_{2}\right), 2.81$ ( $\left.\mathrm{t}, 2 \mathrm{H}, \mathrm{CH}_{2}\right), 6.80-7.21$ (m, 5H, Ar-H and $\left.\mathrm{N}-\mathrm{H}_{\text {Diazep. }}\right), 8.95$ (brs, $\mathrm{H},=\mathrm{NH}, \mathrm{D}_{2} \mathrm{O}$-exchangeable); $\mathrm{MS}(\mathrm{m} / \mathrm{z}$, \%): $333.10\left(\mathrm{M}^{+}, 25\right)$; Anal. Calcd. for $\mathrm{C}_{19} \mathrm{H}_{15} \mathrm{~N}_{3} \mathrm{O}_{3}$ (333.34): $\mathrm{C}, 68.46 ; \mathrm{H}, 4.54 ; \mathrm{N}, 12.61 \%$. Found: $\mathrm{C}$, $68.18 ; \mathrm{H}, 4.24 ; \mathrm{N}, 12.31 \%$. 
14-Methyl-2,3-dihydrobenzo[b]cyclopenta[ $\left[5^{\prime}, 6^{\prime}\right]$ pyrano $\left[4^{\prime}, 3^{\prime}: 4,5\right]$ pyrano[2,3-e] $[1,4]$ diazepine-5,6(1H,8H)-dione (32). A mixture of imino analogue $31(0.33 \mathrm{~g}, 1 \mathrm{mmol}), \mathrm{HCl}_{\text {conc }}$. $(2 \mathrm{~mL})$ in $\mathrm{EtOH}(30 \mathrm{~mL})$ was refluxed for $1 \mathrm{~h}$. After cooling the mixture was poured onto mashed ice. A yellow precipitate was collected, washed with $\mathrm{H}_{2} \mathrm{O}$ carefully, dried, and recrystallized using EtOH to furnish 32 with an $85 \%$ yield, mp 238-240 ${ }^{\circ} \mathrm{C}$; IR (KBr): $\left(\mathrm{cm}^{-1}\right) 1610\left(\mathrm{C}=\mathrm{N}_{\text {str. }}\right), 1705\left(2 \mathrm{C}=\mathrm{O}_{\text {str }}\right), 3256\left(\mathrm{~N}-\mathrm{H}_{\text {str. }}\right){ }^{1} \mathrm{H} \mathrm{NMR}(300 \mathrm{MHz}$, DMSO- $\left.d_{6}\right): \delta 1.83\left(\mathrm{~m}, 2 \mathrm{H}, \mathrm{CH}_{2}\right), 2.06(\mathrm{~s}, 3 \mathrm{H}, \mathrm{Me}), 2.43\left(\mathrm{t}, 2 \mathrm{H}, \mathrm{CH}_{2}\right), 2.81\left(\mathrm{t}, 2 \mathrm{H}, \mathrm{CH}_{2}\right), 6.80-7.21(\mathrm{~m}, 5 \mathrm{H}$, Ar-H and N-H $\left.\mathrm{H}_{\text {Diazep. }}\right) ;{ }^{13} \mathrm{C}$ NMR $\left(75 \mathrm{MHz}\right.$, DMSO- $\left.d_{6}\right)$ : $26.6(2 \mathrm{Me}), 18.6,36.1,36.6\left(3 \mathrm{CH}_{2}\right), 76.6,113.5$, 116.1, 118.5, 123.5, 124.1, 126.5, 128.5, 137.2, 138.1, 140.6, 170.1 (12 C=C), 162.7, 162.9 (2 C=O), 164.6 $(\mathrm{C}=\mathrm{N})$; $\mathrm{MS}(\mathrm{m} / \mathrm{z}, \%)$ : $334.10\left(\mathrm{M}^{+}, 30\right)$; Anal. Calcd. for $\mathrm{C}_{19} \mathrm{H}_{14} \mathrm{~N}_{2} \mathrm{O}_{4}$ (334.33): $\mathrm{C}, 68.26 ; \mathrm{H}, 4.22 ; \mathrm{N}, 8.38 \%$. Found: $\mathrm{C}, 68.12 ; \mathrm{H}, 4.01 ; \mathrm{N}, 8.15 \%$.

1-Acetyl-2,13-dimethyl-4H-benzo[b]pyrano[4' $\left.3^{\prime}: 4,5\right]$ pyrano[2,3-e][1,4]diazepine-4,5(7H)-dione

A mixture of compound $5(0.29 \mathrm{~g}, 1 \mathrm{mmol})$ and acetylacetone $(0.10 \mathrm{~mL}, 1 \mathrm{mmol})$ was refluxed in ethanolic-piperidine solution $(25 \mathrm{~mL})$ for $6 \mathrm{~h}$. Upon cooling the reaction mixture an off-white precipitate was collected, and recrystallized using $\mathrm{EtOH}$ to give 33 with an $80 \%$ yield, $\mathrm{mp} 216-218{ }^{\circ} \mathrm{C}$; IR (KBr): $\left(\mathrm{cm}^{-1}\right) 1610\left(\mathrm{C}=\mathrm{N}_{\text {str. }}\right), 1705\left(2 \mathrm{C}=\mathrm{O}_{\text {str. }}\right), 3206\left(\mathrm{~N}-\mathrm{H}_{\text {str. }}\right) ;{ }^{1} \mathrm{H}$ NMR (300 MHz, DMSO- $\left.d_{6}\right): \delta 2.06$, $2.53(\mathrm{~m}, 6 \mathrm{H}, 2 \mathrm{Me}), 2.36$ (s, 3H, COMe), 6.80-7.21 (m, 5H, Ar-H and N-H Diazep.); MS ( $\mathrm{m} / \mathrm{z}, \%): 350.09$ $\left(\mathrm{M}^{+}, 10\right)$; Anal. Calcd. for $\mathrm{C}_{19} \mathrm{H}_{14} \mathrm{~N}_{2} \mathrm{O}_{5}$ (350.32): $\mathrm{C}, 65.14 ; \mathrm{H}, 4.03 ; \mathrm{N}, 8.00 \%$. Found: $\mathrm{C}, 65.01 ; \mathrm{H}, 3.86$; $\mathrm{N}, 7.78 \%$.

13-Methyl-1-thioxo-1,2-dihydro-[1,3,5]triazino[1", 2" $\left.: 1^{\prime}, 5^{\prime}\right]$ pyrazolo[ $\left.3^{\prime}, 4^{\prime}: 4,5\right]$ pyrano[2,3-e]benzo[b][1,4] diazepin-5(7H)-one (34). A solution of $\alpha$-aminocarbothiamide $9(0.34 \mathrm{~g}, 1 \mathrm{mmol})$ and $\mathrm{HC}(\mathrm{OEt})_{3}(10 \mathrm{~mL})$ was refluxed for $6 \mathrm{~h}$. The mixture was cooled, a yellow solid was obtained, then collected by filtration, dried, and recrystallized using EtOH to give 34 with a $76 \%$ yield; $\mathrm{mp} 202-204^{\circ} \mathrm{C}$; IR $(\mathrm{KBr}):\left(\mathrm{cm}^{-1}\right) 1326$ $\left(\mathrm{C}=\mathrm{S}_{\text {str. }}\right), 1610-1623\left(3 \mathrm{C}=\mathrm{N}_{\text {str. }}\right), 1705\left(\mathrm{C}=\mathrm{O}_{\text {str. }}\right), 3206-3325\left(2 \mathrm{~N}-\mathrm{H}_{\text {str. }}\right) ;{ }^{1} \mathrm{H}$ NMR $\left(300 \mathrm{MHz}, \mathrm{DMSO}-d_{6}\right)$ : $\delta 2.07(\mathrm{~s}, 3 \mathrm{H}, \mathrm{Me}), 6.80-7.95\left(\mathrm{~m}, 6 \mathrm{H}, \mathrm{Ar}-\mathrm{H}\right.$, Triazi.(C6) $-1 \mathrm{H}$, and $\left.\mathrm{N}-\mathrm{H}_{\text {Diazep. }}\right), 13.56$ (brs, $\mathrm{H}, \mathrm{NH}_{\text {Triazi }}$, $\mathrm{D}_{2} \mathrm{O}$-exchangeable); $\mathrm{MS}(\mathrm{m} / \mathrm{z}, \%): 350.05\left(\mathrm{M}^{+}, 42\right)$; Anal. Calcd. for $\mathrm{C}_{16} \mathrm{H}_{10} \mathrm{~N}_{6} \mathrm{O}_{2} \mathrm{~S}$ (350.35): $\mathrm{C}, 54.85 ; \mathrm{H}$, $2.88 ; \mathrm{N}, 23.99 \%$. Found: $\mathrm{C}, 54.51 ; \mathrm{H}, 2.49 ; \mathrm{N}, 23.64 \%$.

3,13-Dimethyl-1-thioxo-1,2-dihydro-[1,3,5]triazino[1",2": $\left.1^{\prime}, 5^{\prime}\right]$ pyrazolo[3' $\left.4^{\prime}: 4,5\right]$ pyrano[2,3-e]benzo[b][1,4] diazepin-5(7H)-one (35). A mixture of $\alpha$-aminocarbothiamide $9(0.68 \mathrm{~g}, 2 \mathrm{mmol})$ and acetyl chloride $(2 \mathrm{mmol})$ was refluxed in benzene $(30 \mathrm{~mL})$ for $6 \mathrm{~h}$. Upon cooling the isolated product was collected and recrystallized using benzene to afford 35 as an off-white powder, with a $70 \%$ yield; mp $182-184{ }^{\circ} \mathrm{C}$; IR (KBr): $\left(\mathrm{cm}^{-1}\right) 1326\left(\mathrm{C}=\mathrm{S}_{\text {str. }}\right), 1610-1623\left(3 \mathrm{C}=\mathrm{N}_{\text {str. }}\right), 1705\left(\mathrm{C}=\mathrm{O}_{\text {str. }}\right), 3206-3325\left(2 \mathrm{~N}-\mathrm{H}_{\text {str. }}\right) ;{ }^{1} \mathrm{H}$ NMR

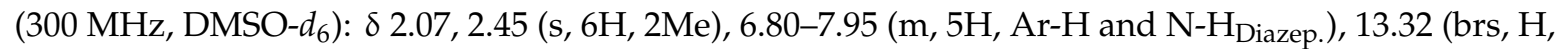
$\mathrm{NH}_{\text {Triazi }}$, $\mathrm{D}_{2} \mathrm{O}$-exchangeable); ${ }^{13} \mathrm{C}$ NMR $\left(75 \mathrm{MHz}\right.$, DMSO- $\left.d_{6}\right): 19.8,21.2(2 \mathrm{Me}), 83.9,113.5,114.4,123.5$, 124.1, 126.5, 128.5, 138.1, 139.2, $147.1(10 \mathrm{C}=\mathrm{C}), 150.4(\mathrm{C}=\mathrm{O}), 133.8,154.3,164.6(3 \mathrm{C}=\mathrm{N}), 189.5(\mathrm{C}=\mathrm{S})$; MS (m/z, \%): $364.07\left(\mathrm{M}^{+}, 15\right)$; Anal. Calcd. for $\mathrm{C}_{17} \mathrm{H}_{12} \mathrm{~N}_{6} \mathrm{O}_{2} \mathrm{~S}$ (364.38): $\mathrm{C}, 56.04 ; \mathrm{H}, 3.32 ; \mathrm{N}, 23.06 \%$. Found: C, 55.85; H, 3.15; N, 22.79\%.

2-(13-Methyl-5-oxo-1-thioxo-1,2,5,7-tetrahydro-[1,3,5]triazino[1",2": $\left.1^{\prime}, 5^{\prime}\right]$ pyrazolo[ $\left[3^{\prime}, 4^{\prime}: 4,5\right]$ pyrano[2,3-e] benzo[b][1,4]diazepin-3-yl)acetonitrile (36). It was synthesized under the same conditions as those described for the synthesis of compound 33. Yellow crystals $(\mathrm{EtOH})$ with a $70 \%$ yield; $\mathrm{mp} 198-200$ ${ }^{\circ} \mathrm{C}$; IR (KBr): $\left(\mathrm{cm}^{-1}\right) 1325\left(\mathrm{C}=\mathrm{S}_{\text {str. }}\right), 1610-1623\left(3 \mathrm{C}=\mathrm{N}_{\text {str. }}\right), 1705\left(\mathrm{C}=\mathrm{O}_{\text {str. }}\right), 2215\left(\mathrm{C} \equiv \mathrm{N}_{\text {str. }}\right), 3206-3325$ $\left(2 \mathrm{~N}-\mathrm{H}_{\text {str. }}\right) ;{ }^{1} \mathrm{H}$ NMR $\left(300 \mathrm{MHz}, \mathrm{DMSO}-d_{6}\right): \delta 2.07(\mathrm{~s}, 3 \mathrm{H}, \mathrm{Me}), 4.17\left(\mathrm{~s}, 2 \mathrm{H}, \mathrm{CH}_{2}\right), 6.80-7.95(\mathrm{~m}, 5 \mathrm{H}, \mathrm{Ar}-\mathrm{H}$ and $\left.\mathrm{N}-\mathrm{H}_{\text {Diazep. }}\right), 13.61$ (brs, $\mathrm{H}, \mathrm{NH}_{\text {Triazi }}$, $\mathrm{D}_{2} \mathrm{O}$-exchangeable); ${ }^{13} \mathrm{C}$ NMR (75 MHz, DMSO- $\left.d_{6}\right): 19.8$ (Me), $22.2\left(\mathrm{CH}_{2}\right), 116.2(\mathrm{C} \equiv \mathrm{N}), 83.9,113.5,114.4,123.5,124.1,126.5,128.5,138.1,139.2,147.1(10 \mathrm{C}=\mathrm{C})$, $150.4(\mathrm{C}=\mathrm{O}), 133.8,154.3,164.6(3 \mathrm{C}=\mathrm{N}), 189.5(\mathrm{C}=\mathrm{S}) ; \mathrm{MS}(\mathrm{m} / \mathrm{z}, \%): 389.05\left(\mathrm{M}^{+}, 35\right)$; Anal. Calcd. for $\mathrm{C}_{18} \mathrm{H}_{11} \mathrm{~N}_{7} \mathrm{O}_{2} \mathrm{~S}$ (389.39): C, 55.52; $\mathrm{H}, 2.85 ; \mathrm{N}, 25.18 \%$. Found: $\mathrm{C}, 55.25 ; \mathrm{H}, 2.63 ; \mathrm{N}, 25.02 \%$.

3-(Chloromethyl)-13-methyl-1-thioxo-1,2-dihydro-[1,3,5]triazino[1",2": $\left.1^{\prime}, 5^{\prime}\right]$ pyrazolo[3' $\left.4^{\prime}: 4,5\right]$ pyrano[2,3-e] benzo[b][1,4]diazepin-5(7H)-one (37). To a well-stirred solution of $\alpha$-aminocarbothiamide 9 (0.68 g, $2 \mathrm{mmol})$ and $\mathrm{Et}_{3} \mathrm{~N}(0.3 \mathrm{~mL})$ in absolute $\mathrm{EtOH}(30 \mathrm{~mL}), \mathrm{ClCH}_{2} \mathrm{COCl}(0.24 \mathrm{~mL}, 1 \mathrm{mmol})$ was added 
dropwise for $1 \mathrm{~h}$ at $\mathrm{rt}$, and afterwards the mixture was warmed for $6 \mathrm{~h}$ at $60{ }^{\circ} \mathrm{C}$. After cooling the reaction mixture and pouring onto mashed ice, a light brown solid was collected by filtration, dried, and recrystallized using EtOH, to furnish 37 with an $80 \%$ yield; mp $198-200{ }^{\circ} \mathrm{C}$; IR $(\mathrm{KBr}):\left(\mathrm{cm}^{-1}\right) 1326$ $\left(\mathrm{C}=\mathrm{S}_{\text {str. }}\right), 1610-1623\left(3 \mathrm{C}=\mathrm{N}_{\text {str. }}\right), 1705\left(\mathrm{C}=\mathrm{O}_{\text {str. }}\right), 3206-3325\left(2 \mathrm{~N}-\mathrm{H}_{\text {str. }}\right) ;{ }^{1} \mathrm{H}$ NMR $\left(300 \mathrm{MHz}, \mathrm{DMSO}-d_{6}\right)$ : $\delta 2.07(\mathrm{~s}, 3 \mathrm{H}, \mathrm{Me}), 3.29\left(\mathrm{~s}, 2 \mathrm{H}, \mathrm{CH}_{2}\right), 6.80-7.95\left(\mathrm{~m}, 5 \mathrm{H}, \mathrm{Ar}-\mathrm{H}\right.$ and N-H Diazep.), 13.63 (brs, $\mathrm{H}, \mathrm{NH}_{\text {Triazi }}$, $\mathrm{D}_{2} \mathrm{O}$-exchangeable); $\mathrm{MS}(\mathrm{m} / \mathrm{z}, \%)$ : $400.05\left(\mathrm{M}^{+}+2,10\right), 398.04\left(\mathrm{M}^{+}, 32\right)$; Anal. Calcd. for $\mathrm{C}_{17} \mathrm{H}_{11} \mathrm{ClN}_{6} \mathrm{O}_{2} \mathrm{~S}$ (398.83): C, 51.20; H, 2.78; Cl, 8.89; N, 21.07\%. Found: C, 51.10; H, 2.56; Cl, 8.58; N, 20.85\%.

3-Mercapto-13-methyl-1-thioxo-1,2-dihydro-[1,3,5]triazino[1",2": $\left.1^{\prime}, 5^{\prime}\right]$ pyrazolo [3',4':4,5]pyrano[2,3-e] benzo [b][1,4]diazepin-5(7H)-one (38). $\mathrm{CS}_{2}$ (4 mmol) was added to solution of $\alpha$-aminocarbothiamide 9 (0.68 g, $2 \mathrm{mmol})$ in $\mathrm{EtOH}(50 \mathrm{~mL})$, afterwards the mixture was heated in water bath at $80^{\circ} \mathrm{C}$ for $6 \mathrm{~h}$. After evaporation of the solvent to one fourth of its volume, the mixture was poured into mashed ice. The formed product was collected, washed carefully with $\mathrm{H}_{2} \mathrm{O}$, and recrystallized using $\mathrm{MeOH}$ to give 38 as a yellowish-brown powder, with a $65 \%$ yield; mp $213-215^{\circ} \mathrm{C}$; $\mathrm{IR}(\mathrm{KBr}):\left(\mathrm{cm}^{-1}\right) 1326\left(\mathrm{C}=\mathrm{S}_{\text {str. }}\right)$, $1610-1623\left(3 \mathrm{C}=\mathrm{N}_{\text {str. }}\right), 1705\left(\mathrm{C}=\mathrm{O}_{\text {str. }}\right), 2453\left(\mathrm{~S}-\mathrm{H}_{\text {str. }}\right), 3206-3325\left(2 \mathrm{~N}-\mathrm{H}_{\text {str. }}\right) ;{ }^{1} \mathrm{H}$ NMR $\left(300 \mathrm{MHz}, \mathrm{DMSO}-d_{6}\right)$ : $\delta 2.07$ (s, 3H, Me), 6.80-7.95 (m, 5H, Ar-H and N-H Diazep.), 12.01 (s, 1H, S-H, $\mathrm{D}_{2} \mathrm{O}$ exchangeable), 13.41 (brs, $\mathrm{H}, \mathrm{NH}_{\text {Triazi., }} \mathrm{D}_{2} \mathrm{O}$-exchangeable); $\mathrm{MS}(\mathrm{m} / \mathrm{z}, \%)$ : $382.42\left(\mathrm{M}^{+}, 35\right)$; Anal. Calcd. for $\mathrm{C}_{16} \mathrm{H}_{10} \mathrm{~N}_{6} \mathrm{O}_{2} \mathrm{~S}_{2}$ (382.42): C, 50.25; H, 2.64; N, 21.98\%. Found: C, 50.14; H, 2.32; N, 21.69\%.

\subsection{Cytotoxic Assessment}

Methodology

The preliminary cytotoxic impacts were achieved using the SRB method as previously reported [30,31].

\section{Conclusions}

The purpose of this study was to synthesize and evaluate the cytotoxic impact of some new benzo[ $b][1,4]$ diazepines. We synthesized 5-methyl- 4-(methylthio)- 2-oxo-2,11- dihydrobenzo[b]pyrano [2,3-e][1,4]diazepine-3-carbonitrile (5), and 3-amino-12-methyl-4-oxo-4,6-dihydro-2 $H$-benzo[ $b]$ pyrazolo $\left[3^{\prime}, 4^{\prime}: 4,5\right]$ pyrano[2,3-e][1,4]diazepine-2-carbothioamide (9), as a new bipod and tripod pharmacophoric architectures. The vitality of the terminal $o$-methylthionitrile as well as $\alpha$-aminocarbothiamide tags were inspected in a sequence of treatments, including cyclocondensation at the annulation of new tri and/or tetrapod pharmacophoric analogues. The preliminary cytotoxicity declared that, majority of the examined compounds own momentous cytotoxic activities. Compound 9 was the most effective among the screened series with $\mathrm{IC}_{50}=16.19 \pm 1.35$ and $17.16 \pm 1.54 \mu \mathrm{M}$ against HCT-116 and MCF-7, respectively. Moreover, the tested tumor cell lines were generally susceptible to conjugates 7, 6, 8, 15, 16, 18, 19, and 20, in a downward order, with $\mathrm{IC}_{50}<60.00 \mu \mathrm{M}$.

Author Contributions: Conceptualization: I.H.E.A.; performing the research: I.H.E.A. and N.A.A.E.; writing—original draft: N.A.A.E.; and review, editing, and supervision: I.H.E.A. All authors have read and agreed to the published version of the manuscript.

Funding: This research received no external funding.

Acknowledgments: This work is dedicated to my father (Helmy El Azab).

Conflicts of Interest: The authors declare no conflict of interest.

\section{References}

1. Verma, S.; Kumar, S.; Kumar, S. Design, synthesis, computational and biological evaluation of new benzodiazepines as CNS agents. Arab. J. Chem. 2020, 13, 863-874. [CrossRef]

2. Sigel, E.; Ernst, M. The Benzodiazepine Binding Sites of GABAA Receptors. Trends Pharmacol Sci. 2018, 39, 659-671. [CrossRef] [PubMed] 
3. Gomez, A.F.; Barthel, A.L.; Hofmann, S.G. Comparing the efficacy of benzodiazepines and serotonergic anti-depressants for adults with generalized anxiety disorder: A meta-analytic review. Expert Opin Pharmacother. 2018, 19, 883-894. [CrossRef] [PubMed]

4. Gustafsson, S.; Lindström, V.; Ingelsson, M.; Hammarlund-Udenaes, M.; Syvänen, S. Intact blood-brain barrier transport of small molecular drugs in animal models of amyloid beta and alpha-synuclein pathology. Neuropharmacology 2018, 128, 482-491. [CrossRef] [PubMed]

5. Chadha, S.; Paul, S.; Kapoor, K.K. Synthesis and biological screening of 4-(5-alkyl-2-isoxazolin-3-yl)2-aryl-2,3-dihydro-1H-1,5-benzodiazepines. J. Chem. Pharm. Res. 2011, 3, 331-340.

6. Sachdeva, A.; Choudhary, M.; Chandra, M. Alcohol withdrawal syndrome: Benzodiazepines and beyond. J. Clin. Diagn. Res. 2015, 9, VE01-VE07. [CrossRef] [PubMed]

7. Xia, J.; Li, J.; Sun, H. Insights into $\mathrm{ET}_{\mathrm{A}}$ subtype selectivity of benzodiazepine endothelin receptor antagonists by 3D-QSAR approaches. J. Mol. Model. 2012, 18, 1299-1311. [CrossRef]

8. Soyka, M. Treatment of benzodiazepine dependence. N. Engl. J. Med. 2017, 376, 1147-1157. [CrossRef]

9. Guina, J.; Merrill, B. Benzodiazepines I: Upping the care on downers: The evidence of risks, benefits and alternatives. J. Clin. Med. 2018, 7, 17. [CrossRef]

10. Manconi, M.; Ferri, R.; Miano, S.; Maestri, M.; Bottasini, V.; Zucconi, M.; Ferini-Strambi, L. Sleep architecture in insomniacs with severe benzodiazepine abuse. J. Clin. Neurophysiol. 2017, 128, 875-881. [CrossRef]

11. Lawrence, P.; Tardibono, Jr.; Marvin, J.M. Synthesis and Anti-Cancer Activity of New Hydroxamic Acid Containing 1,4-Benzodiazepines. Org. Lett. 2009, 11, 1575-1578.

12. Menghani, S.S.; Chikhale, R.; Pant, A.; Mathew, B.; Khedekar, P. Molecular Docking, Synthesis and CNS Activity of Some Novel 1,4-Benzodiazepine Derivatives. Lett. Drug Des. Discovery 2017, 14, 690-698. [CrossRef]

13. Griffin, C.E.; Kaye, A.M.; Bueno, F.R.; Kaye, A.D. Benzodiazepine pharmacology and central nervous system-mediated effects. Ochsner J. 2013, 13, 214-223. [PubMed]

14. Kumaraswamy, S.; Chien-Shu, C.; Chi-Fen, C.; Srinivas, P.; Khagga, M.; Chi-Rei, W.; Ta-Hsien, C. Synthesis, Anticonvulsant, Sedative and Anxiolytic Activities of Novel Annulated Pyrrolo[1,4]benzodiazepines. Int. J. Mol. Sci. 2014, 15, 16500-16510.

15. Włodarczyk, A.; Szarmach, J.; Cubała, W.J.; Wiglusz, M.S. Benzodiazepines in combination with antipsychotic drugs for schizophrenia: GABA-ergic targeted therapy. Psychiatr Danub. 2017, 29, 345-348.

16. Mahmoud, A.A.; El-Sayed, W.M. The Anti-Proliferative Activity of Anisosciadone: A New Guaiane Sesquiterpene from Anisosciadium lanatum. Anti-Cancer Agents Med. Chem. 2019, 19, 1114-1119. [CrossRef]

17. Zhang, Q.; Shen, Q.; Gao, L.; Tong, L.; Li, J.; Chen, Y.; Lu, W. Pyrazolo[4,3-b]pyrimido[4,5-e] [1,4]diazepine derivatives as new multi-targeted inhibitors of Aurora A/B and KDR. Eur. J. Med. Chem. 2018, 5, 428-441. [CrossRef]

18. Bader, A.A.S.; Omar, A.H.; El-Odemi, M.H. Anti-inflammatory Effects of Diazepam on Different Models of Inflammation: Roles of Peripheral Benzodiazepine Receptors and Genes for Corticosterone, Nitric Oxide and Cytokines Biosynthesis. J. Clin. Epigenet. 2017, 3, 1-8.

19. Jing-Jing, L.; Xin-Ying, W.; Jing-Lou, C.; Guan-Rong, C.; Jun, X.; Ye, G.; Hong-Ping, S. Antiplatelet drug ticagrelor delays gastric ulcer healing in rats. Exp. Ther. Med. 2017, 14, 3774-3779.

20. Pavlovsky, V.I.; Tsymbalyuk, O.V.; Martynyuk, V.S.; Kabanova, T.A.; Semenishyna, E.A.; Khalimova, E.I.; Andronati, S.A. Analgesic Effects of 3-Substituted Derivatives of 1,4-Benzodiazepines and their Possible Mechanisms. Neurophysiology 2013, 45, 427-432. [CrossRef]

21. El Azab, I.H.; Elkanzi, N.A.A. Synthesis and anti-tumour activity of some new sebacoyl chloride-based heterocycles. Curr. Org. Synth. 2017, 14, 309-320. [CrossRef]

22. El Azab, I.H.; Elkanzi, N.A.A. Design, Synthesis, and Antimicrobial Evaluation of New Annelated Pyrimido[2,1-c][1,2,4]triazolo[3,4-f][1,2,4]triazines. Molecules 2020, 25, 1339. [CrossRef] [PubMed]

23. El Azab, I.H.; Elkanzi, N.A.A.; Gobouri, A.A. Design and Synthesis of Some New Quinoxaline-Based Heterocycles. J. Heterocycl. Chem. 2018, 55, 65-76. [CrossRef]

24. El Azab, I.H.; Gobouri, A.A.; Altalhi, T.A. 4-Chlorothiazole-5-carbaldehydes as Potent Precursors for Synthesis of Some New Pendant N-heterocyces Endowed with Anti-Tumor Activity. J. Heterocycl. Chem. 2019, 56, 281-295. [CrossRef] 
25. El Azab, I.H.; Elkanzi, N.A.A.; Gobouri, A.A.; Altalhi, T.A. Convenient Synthesis of Novel Nitrogen Bridgehead Heterocycles Utilizing 3-Mercapto-6H-[1,2,4,5]oxatriazino[3,2- $a]$ isoindol-6-one as a New Synthon. J. Heterocycl. Chem. 2019, 56, 60-72. [CrossRef]

26. El Azab, I.H.; Abu Ali, O.A.; El-Zahrani, A.H.; Gobouri, A.A.; Altalhi, T.A. Pyrazole-1-carbothioamide as a Potent Precursor for Synthesis of Some New N-heterocycles of Potential Biological Activity. J. Heterocycl. Chem. 2019, 56, 18-31. [CrossRef]

27. El Azab, I.H.; Khalifa, M.E.; Gobouri, A.A.; Altalhi, T.A. Synthesis, Characterization, and Pharmacological Evaluation of Some New Pteridine-Based Heterocycles as Antimicrobial Agents. J. Heterocycl. Chem. 2019, 56, 1352-1361. [CrossRef]

28. Israel, M.; Jones, L.C.; Modest, E.J. Thermal rearrangement of condensed dihydrodiazepinones. Tetrahedron Lett. 1968, 46, 4811-4814. [CrossRef]

29. Maurya, H.K.; Vasudev, A.; Gupta, A. A regioselective synthesis of 2,6-diarylpyridines. RSC Adv. 2013, 3, 12955-12962. [CrossRef]

30. Youns, M.; Fu, Y.J.; Zu, Y.G.; Kramer, A.; Konkimalla, V.B.; Radlwimmer, B.; Sultmann, H.; Efferth, T. Sensitivity and resistance towards isoliquiritigenin, doxorubicin and methotrexate in $\mathrm{T}$ cell acute lymphoblastic leukaemia cell lines by pharmacogenomics. Arch. Pharmacol. 2010, 382, 221-234. [CrossRef]

31. Kuete, V.; Krusche, B.; Youns, M.; Voukeng, I.; Fankam, A.G.; Tankeo, S.; Lacmata, S.; Efferth, T. Cytotoxicity of some Cameroonian spices and selected medicinal plant extracts. J. Ethnopharmacol. 2011, 134, 803-812. [CrossRef] [PubMed]

Sample Availability: Samples of the compounds 6, 7, 8, 9, 15, 16, 18, 19 and 20 are available from the authors.

(C) 2020 by the authors. Licensee MDPI, Basel, Switzerland. This article is an open access article distributed under the terms and conditions of the Creative Commons Attribution (CC BY) license (http://creativecommons.org/licenses/by/4.0/). 